\title{
REFLECTION GROUPS ON RIEMANNIAN MANIFOLDS
}

\author{
DMITRI ALEKSEEVSKY, ANDREAS KRIEGL, MARK LOSIK, PETER W. MICHOR
}

\begin{abstract}
We investigate discrete groups $G$ of isometries of a complete connected Riemannian manifold $M$ which are generated by reflections, in particular those generated by disecting reflections. We show that these are Coxeter groups, and that the the orbit space $M / G$ is isometric to a Weyl chamber $C$ which is a Riemannian manifold with corners and certain angle conditions along intersections of faces. We can also reconstruct the manifold and its action from the Riemannian chamber and its equipment of isotropy group data along the faces. We also discuss these results from the point of view of Riemannian orbifolds.
\end{abstract}

\section{INTRODUCTION}

The aim of this paper is to study the discrete groups $G$ generated by reflections with respect to hypersurfaces (shortly, reflection groups ) on a Riemannian manifold $M$. If $M=E^{n}$ is the Euclidean space, then the classification of all reflection groups was given in a fundamental paper by Coxeter [11]. This implies also the classification of reflection groups on the sphere $S^{n}$. There are many results about reflection groups in hyperbolic space, see Vinberg [32], [34], [33], and [35], but the complete classification is missing. In all these cases the appropriate fundamental domain $C$ of a reflection group $G$ (called Weyl chamber) is a Coxeter polyhedron, i.e., a convex polyhedron where any two neighbour walls (codimension 1 faces $F_{i}$, $F_{j}$ with codimension 2 intersection) have angle $\pi / n_{i, j}$ for $n_{i, j} \in \mathbb{N}$. We call this the Coxeter property. Conversely, any Coxeter polyhedron $C$ in a space of constant curvature $M=S^{n}, E^{n}, H^{n}$ is the fundamental domain of the reflection group $G$ which is generated by the reflections $s_{i}=s_{F_{i}}$ with respect to the walls $F_{i}$ of $C$. The group $G$ is a Coxeter group, i.e., a group with a set $S=\left\{s_{1}, \ldots, s_{l}\right\}$ of generators, and relations $s_{i}^{2}=1,\left(s_{i} s_{j}\right)^{n_{i, j}}=1$ for $n_{i, j} \in \mathbb{N} \cup\{\infty\}$. In our case, $n_{i, j}$ is defined by the angle between the walls $F_{i}$ and $F_{j}$ as above.

The manifold $M$ with the action of $G$ can be reconstructed from the Weyl chamber $C$ (which is homeomorphic to the orbit space $M / G$ ) by the universal construction of Vinberg [32]: Define the equivalence relation in $G \times C$ by

$$
(x, g) \sim(y, h) \Longleftrightarrow x=y, g^{-1} h \in G_{x}
$$

where $G_{x}=\left\langle s_{F_{i}}: x \in F_{i}\right\rangle$ is the subgroup generated by all reflections with respect to walls containing $x$. Then the quotient space

$$
\mathcal{U}(G, C)=G \times C / \sim
$$

2000 Mathematics Subject Classification. Primary 51F15, 53C20, 20F55, 22 E40.

Key words and phrases. Reflection groups, Isometries.

P.W.M. and M.L. were supported by 'Fonds zur Förderung der wissenschaftlichen Forschung, Projekt P 14195 MAT'. 
has the structure of a space of constant curvature such that the natural action of $G$ on $\mathcal{U}(G, C)=M$ is isometric and $G$ is the reflection group of $M$ with fundamental domain $C$.

More generally, if $G$ is a Coxeter group with a set $S=\left\{s_{1}, \ldots, s_{l}\right\}$ of standard generators, and relations $s_{i}^{2}=1,\left(s_{i} s_{j}\right)^{n_{i, j}}=1$, where $i, j=1, \ldots, l$ and $n_{i j} \in$ $\mathbb{N} \cup\{\infty\}$, and if $C$ is a topological space with closed subspaces $P_{1}, \ldots, P_{l}$ (called panels), then the Vinberg construction with $G_{x}=\left\langle s_{i}: x \in P_{i}\right\rangle$ gives a topological space $\mathcal{U}(G, C)$ with a continuous action of the group $G$ and orbit space $C$. The topological $G$-space $\mathcal{U}(G, C)$ is called the universal space of the Coxeter group $G$, and it satisfies the following following universal property [32]:

If $G$ acts in a topological space $X$ and if $\varphi: C \rightarrow X$ is a continuous map such that $s_{i} \cdot \varphi(x)=\varphi(x)$ for $x \in P_{i}$ then there exists a unique extension of $\varphi$ to a $G$-equivariant continuous map $\tilde{\varphi}: \mathcal{U}(G, C) \rightarrow X$ such that $\tilde{\varphi}[1, x]=\varphi(x)$ for any $x \in C$.

Davis [12] found necessary and sufficient conditions that $\mathcal{U}(G, C)$ is a topological manifold and $G$ is a topological reflection group of $\mathcal{U}(G, C)$, i.e., any generator $s_{i}$ acts on $\mathcal{U}(G, C)$ as a topological reflection (an involutive transformation whose fixed point set $\mathcal{U}(G, C)^{s_{i}}$ separates $\left.\mathcal{U}(G, C)\right)$. These conditions are that $C$ is a topological 'nice' manifold with corners and that each panel $P_{i}$ is a disjoint union of walls such that for any $x \in C$ the subgroup $G_{x}=\left\langle s_{i}: x \in P_{i}\right\rangle$ is finite. Conversely, let $G$ be a discrete group of transformations of a topological manifold $M$ generated by topological reflections, and let $C$ be its Weyl chamber (the closure of a connected component of the set $M_{\text {reg }}=\left\{x \in M: G_{x}=\{1\}\right\}$ of regular points). Let $s_{1}, \ldots s_{l}$ be reflections in $G$ such that $M^{s_{i}} \cap C$ contains a codimension 1 component. Let $P_{i}$ be the union of all codimension 1 components $M^{s_{i}} \cap C$. Then $G$ is a Coxeter group with standard generators $s_{1}, \ldots, s_{l}$ and the $G$-manifold $M$ is $G$-homeomorphic to the universal $G$-manifold $\mathcal{U}(G, C)$ defined by the panels $P_{1}, \ldots, P_{l}$.

One of the aims of this paper is to describe the structure of the Weyl chamber $C \cong M / G$ of a Riemannian manifold $M$ with a discrete group $G$ generated by reflections, and to get a similar description of such $G$-manifolds $M$ in terms of 'abstract Riemannian chambers' $C$, which are Riemannian manifolds with corners such that any two neighbouring walls $F_{i}, F_{j}$ satisfy the Coxeter property, i.e., the corresponding angle has constant value $\pi / n_{i j}$ along $F_{i} \cap F_{j}$.

In section (2) we fix terminology and describe general properties of reflections of a Riemannian manifold $M$ and of a discrete group $G$ generated by reflections. We discuss the relations between a Dirichlet domain $D$ of the group $G$ and its Weyl chamber $C$ which is defined as the closure of a connected component of the set $M_{\text {reg }}$ of regular points of $G$. We give an example when a Weyl chamber is larger than a Dirichlet domain. We prove that for a simply connected manifold $M$, any reflection $s$ is disecting, i.e. its fixed point set $M^{s}$ is a connected totally geodesic hypersurface which decomposes $M$ into two parts. We observe that a reflection group $G$ on a Riemannian manifold $M$ can be lifted canonically to a reflection group $\tilde{G}$, which is an extension of $G$, on the universal covering $\tilde{M}$ of $M$. As an interesting example of Riemannian manifold with a group generated by non disecting reflections, we consider the maximal torus of the group $S U(n)$ for $n>2$ with the action of the Weyl group.

Starting from section (3), we mostly consider a Riemannian manifold $M$ with a reflection group $G$ generated by disecting reflections. Such a $G$-manifold is called 
a Coxeter manifold. Following M. Davis [12], we derive from a lemma of Bourbaki [4] that the group $G$ acts simply transitively on the set of Weyl chambers $C$ of a Coxeter $G$-manifold $M$. This implies that Weyl chambers coincide with Dirichlet domains of regular points and hence are homeomorphic to the orbit space, and that the reflection group $G$ is a Coxeter group with reflections $s_{i}$ with respect to walls $F_{i}$ of $C$ as standard generators. Moreover, the Weyl chamber $C$ has the structure of a Riemannian manifold with corners and any two neighbouring walls $F_{i}, F_{j}$ of $C$ satisfy the Coxeter property and yield a Coxeter relation $\left(s_{i} s_{j}\right)^{n_{i j}}=1$. We prove that in the simply connected case these relations generate all relations of $G$. In the general case, we give a geometric description of the fundamental group $\pi_{1}(M)$.

In section (4) we recall the notion and main the properties of a (smooth) manifold $M$ with corners and we define the concept of a Coxeter equipment of $M$. This is an order reversing mapping of the poset of faces of $M$ into the poset of Coxeter subgroups of a given Coxeter system $(G, S)$ (where $S$ is the set of standard generators of a Coxeter group $G$ ) which satisfies the Vinberg finiteness condition, see [34], [13]. We define a notion of Riemannian chamber $C$ as a manifold with corners $C$ equipped with an appropriate Riemannian metric such that walls $W_{i}$ of $C$ are totally geodesic and neighbouring walls satisfy the Coxeter property. Any Riemannian chamber carries a universal Coxeter equipment.

The Weyl chamber $C$ of a Coxeter $G$-manifold $M$ has the natural structure of a Riemannian chamber with an admissible (in some rigorous sense) Coxeter equipment. Moreover, this equipment is universal if and only if $\pi_{1}(M)=\pi_{1}(C)$. Conversely, if $C$ is a Riemmanian chamber with an admissible Coxeter $(G, S)$ equipment then the universal space $M=\mathcal{U}(G, C)$ has the structure of a Coxeter $G$-manifold with Weyl chamber $C$. We prove also that if $C$ is a manifold with corners and $s$ is a Coxeter equipment of $M$ then there exist a Riemannian metric $\gamma$ such that $(M, \gamma)$ is a Riemannian chamber and the equipment $s$ is admissible. Hence any manifold with corners $C$ with a Coxeter equipment determines a Coxeter $G$-manifold $M$, where the metric of $M$ depends on the admissible metric on $C$ and any Coxeter manifold can be obtained by this construction.

In section (5) and (6) we discuss another approach for reconstructing the Coxeter manifold from its Weyl chamber $C$ which can be identified with the orbit space $M / G$ based on the Thurston construction [31] of the universal covering orbifold. We recall this construction in section (5) and we derive from the main theorem of [23] that an orbifold structure of a space $X$ can be reconstructed from the sheaf $S_{X}$ of its smooth functions. In section (6) we define the notion of a Coxeter orbifold as an orbifold whose local groups are finite linear Coxeter groups. An example of Coxeter orbifold is the Weyl chamber $C$ of a Coxeter manifold $M$. We prove that any Coxeter orbifold is such a Weyl chamber. More precisely, the universal covering $M=\tilde{C}$ of a Coxeter orbifold $C$ admits a structure of (smooth) Coxeter $G$ manifold such that $C$ is isomorphic to the Weyl chamber of the isometry group $G$. In particular, this shows that any Coxeter orbifold is good in the sense of Thurston.

In the last section we described all Coxeter equipments of an $n$-simplex $\Delta_{n}$. This gives a classification of Coxeter orbifold structures on $\Delta$ and a classification of Coxeter manifolds with orbit space $\Delta_{n}$ up to a diffeomorphism. 


\section{Groups of isometries GenerAted By REFLECTIONS}

2.1 Lemma. Let $M$ be a connected complete Riemannian manifold, and let $G \subseteq$ $\operatorname{Isom}(M)$ be a group of isometries. Then $G$ is a discrete subgroup in the Lie group $\operatorname{Isom}(M)$ if and only if each orbit of $G$ in $M$ is discrete.

We shall say that $G$ acts discretely on $M$.

Proof. The pointwise-open topology on the Lie group $\operatorname{Isom}(M)$ of all isometries coincides with the compact open topology.

If $G$ is a discrete subgroup in $\operatorname{Isom}(M)$ then it is closed and acts properly on $M$ so the action admits slices, and the orbit $G . x$ through $x \in M$ is homeomorphic to $G / G_{x}$ where $G_{x}$ is the isotropy group of $x$. Thus each orbit is discrete.

Conversely, suppose that each orbit is discrete. Since $G$ consists of isometries, each discrete orbit is closed. We consider the closure $\bar{G}$ of $G$ in $\operatorname{Isom}(M)$. Since $G$-orbits are closed, $\bar{G} . x=G . x$ for each $x \in M$. The action of the closed group $\bar{G}$ of isometries is proper, so there exist slices. Let $x_{0}$ be a regular point for the $\bar{G}$-action. Since $\bar{G} \cdot x_{0}$ is discrete, the slice $S_{x_{0}}$ through $x_{0}$ is open in $M$, and the isotropy group $\bar{G}_{x_{0}}$ acts trivial on $S_{x_{0}}$. Thus $\bar{G}_{x_{0}}$ acts trivial on $M$ and equals $\{e\}$. Then $G \cdot x_{0}=\bar{G} \cdot x_{0} \cong \bar{G}$, thus $\bar{G}=G$ and is discrete in $\operatorname{Isom}(M)$.

2.2. Dirichlet domains and central hypersurfaces. Let $G \subset \operatorname{Isom}(M)$ be a group which acts isometrically and discretely on a connected complete Riemannian manifold. Let $x_{0}$ be a regular point. The closed Dirichlet domain for this point is the set

$$
D\left(x_{0}\right):=\left\{y \in M: d\left(y, x_{0}\right) \leq d\left(y, g . x_{0}\right) \text { for all } g \in G\right\},
$$

where $d$ is the geodesic distance on $M$. The open interior $D\left(x_{0}\right)^{o}$ is called the open Dirichlet domain for the regular orbit $G . x_{0}$, and we can find a fundamental domain $F$ for the action of $G$ satisfying $D\left(x_{0}\right)^{o} \subseteq F \subset D\left(x_{0}\right)$, i.e., a set $F$ which meets each orbit in exactly one point, since

$$
M=\bigcup_{g \in G} g \cdot D\left(x_{0}\right) .
$$

For any two different points $y_{0}, y_{1} \in M$ the central hypersurface is given by

$$
H_{y_{0}, y_{1}}:=\left\{y \in M: d\left(y, y_{0}\right)=d\left(y, y_{1}\right)\right\} .
$$

It disects $M$ in the sense that $M \backslash H_{y_{0}, y_{1}}$ is the disjoint union of the two open sets $\left\{x \in M: d\left(x, y_{0}\right)>d\left(x,, y_{1}\right)\right\}$ and $\left\{x \in M: d\left(x, y_{0}\right)<d\left(x,, y_{1}\right)\right\}$. Note that if $M$ is a simply connected space of constant curvature then $H_{y_{0}, y_{1}}$ is a totally geodesic submanifold, since it is the fixed point set of a symmetry, but that in general $H_{y_{0}, y_{1}}$ is not a submanifold: On an elongated 2-torus it can be a figure 8 .

Lemma. For $x \in H_{y_{0}, y_{1}}$ let $c_{0}$ be a minimal geodesic from $y_{0}$ to $x$. Then $c_{0}$ meets $H_{y_{0}, y_{1}}$ only at $x$.

Proof. Let $c_{0}\left(t_{x}\right)=x$ and suppose for contradiction that $c_{0}(t) \in H_{y_{0}, y_{1}}$ for $t<t_{x}$. Let $c_{2}$ be a minimal geodesic from $x$ to $y_{1}$. Then $t_{x}=d\left(y_{0}, c_{0}(t)\right)+$ $d\left(c_{0}(t), x\right)=d\left(y_{1}, c_{0}(t)\right)+d\left(c_{0}(t), x\right)<d\left(y_{1}, x\right)=t_{x}$ unless $c_{2}$ equals the minimal geodesic $s \mapsto c_{1}(t-s)$ and hence $y_{0}=y_{1}$, both a contradiction.

2.3. Lemma. Let $D=D\left(x_{0}\right)$ be the closed Dirichlet domain of a regular point $x_{0}$ for a discrete action of a group $G \subset \operatorname{Isom}(M)$. Then we have:

(1) If $g . D=D$ then $g=e$ in $G$. 
(2) The open Dirichlet domain $D\left(x_{0}\right)^{\circ}$ is the connected component containing $x_{0}$ of

$$
M \backslash \bigcup_{e \neq g \in G} H_{x_{0}, g \cdot x_{0}} \subset M_{r e g} .
$$

Here $M_{\text {reg }}$ denotes the set of all regular points, i.e. those points with trivial stabilizers.

(3) $G$ acts simply transitively on the set $\left\{D\left(g \cdot x_{0}\right): g \in G\right\}$ of all Dirichlet domains.

Proof. The isotropy group $G_{x_{0}}$ is trivial: See the proof of (2.1).

(1) If $g . D=D$ then $g \cdot x_{0} \in D^{o}$, thus $d\left(g \cdot x_{0}, x_{0}\right) \leq d\left(g \cdot x_{0}, h \cdot x_{0}\right)$ for each $h \neq e$ in $G$. If $g \neq e$, putting $h=g$, we get $g \cdot x_{0}=x_{0}$, a contradiction.

(2) If $x \notin \bigcup_{e \neq g \in G} H_{x_{0}, g \cdot x_{0}}$ then $d\left(x, x_{0}\right) \neq d\left(x, g \cdot x_{0}\right)$ for each $e \neq g \in G$. So if $g \cdot x=x$ for $g \neq e$ then $d\left(x, x_{0}\right)=d\left(g \cdot x, x_{0}\right)=d\left(x, g^{-1} \cdot x_{0}\right) \neq d\left(x, x_{0}\right)$, a contradiction. Thus the isotropy group $G_{x}$ is trivial and $x$ is regular. The connected component of $M \backslash \bigcup_{e \neq g \in G} H_{x_{0}, g \cdot x_{0}}$ containing $x_{0}$ is the set of all $x \in M$ with $d\left(x, x_{0}\right)<d\left(x, g . x_{0}\right)$ for all $e \neq g \in G$ which is $D^{o}$.

(3) Transitivity was seen in (2.2) and simple transitivity follows from (1).

2.4. Walls of Dirichlet domains. Let $G \subset \operatorname{Isom}(M)$ be a discrete subgroup. For a regular point $x_{0} \in M$ the set $F:=H_{x_{0}, g \cdot x_{0}} \cap D\left(x_{0}\right)$ is called a wall of the closed Dirichlet domain $D\left(x_{0}\right)$ if it contains an open non-empty subset of $H_{x_{0}, g \cdot x_{0}}$.

Two closed Dirichlet domains are called neighbors if they contain a common wall.

2.5. Lemma of Poincaré. Let $D=D\left(x_{0}\right)$ be a closed Dirichlet domain of a regular point $x_{0}$, and let $g_{1} . D, g_{2} . D, \ldots$ be all the neighbors of $D$. Then the elements $g_{1}, g_{2}, \ldots$ generate the group $G$.

See fig. 4 for a Dirichlet domain with countably many walls.

Proof. Claim. For each $g \in G$ there exists a sequence $e=h_{0}, h_{1}, \ldots, h_{n}=g$ such that $D\left(h_{i} \cdot x_{0}\right)$ and $D\left(h_{i+1} \cdot x_{0}\right)$ are neighbors for each $i$. We call this a Dirichlet neighbors chain from $x_{0}$ to $g \cdot x_{0}$.

The claim proves the lemma as follows. Since $D\left(h_{1} . x_{0}\right)$ is a neighbor of $D=$ $D\left(x_{0}\right)$ we have $D\left(h_{1} \cdot x_{0}\right)=g_{i_{1}} . D$ for some $i_{1}$. Then $g_{i_{1}} \cdot g_{i_{2}} . D$ is the neighbor $D\left(h_{2} . x_{0}\right)$ of $g_{i_{1}} . D$. Finally $g_{i_{1}} \ldots g_{i_{n}} . D$ is the neighbor $D\left(h_{n} . x_{0}\right)=D\left(g . x_{0}\right)$ of $g_{i_{1}} \ldots g_{i_{n-1}} . D=D\left(h_{n-1} \cdot x_{0}\right)$. By $(2.3)$ we have $g=g_{i_{1}} \ldots g_{i_{n}}$.

We prove the claim by induction on $\left\{d_{g}:=d\left(x_{0}, g . x_{0}\right): g \in G\right\}$ which is a locally finite set in $\mathbb{R}$ since the orbit G. $x_{0}$ is discrete and closed in $M$.

Let $g \in G$ and assume that there exists a Dirichlet neighbors chain from $x_{0}$ to $h . x_{0}$ whenever $d_{h}<d_{g}$. Applying $g_{1}$ we then also conclude that there exists a Dirichlet neighbors chain from $g_{1} . x_{0}$ to $g_{2} . x_{0}$ whenever $d\left(g_{1} . x_{0}, g_{2} . x_{0}\right)<d_{g}$. Consider a minimal geodesic $c$ from $x_{0}$ to $g . x_{0}$ of length $d_{g}$.

Case 1. Suppose that $c$ meets $\bigcup_{e \neq k \in G} H_{x_{0}, k . x_{0}}$ in $x=c\left(t_{1}\right) \in H_{x_{0}, k . x_{0}}$ at distance $t_{1}<\frac{1}{2} d_{g}$. See fig. 1. Then $k \neq g$. Consider a minimal geodesic $c_{1}$ from $x_{0}$ to $k \cdot x_{0}$. Then $c_{1}$ meets $H_{x_{0}, k . x_{0}}$ in $c_{1}\left(\frac{1}{2} d_{k}\right)$. The minimal geodesic $c_{2}$ from $x$ to $k . x_{0}$ has length $t_{1}$. Thus $d_{k} \leq 2 t_{1}<d_{g}$ by the triangle inequality. By induction there exists a Dirichlet neighbors chain from $x_{0}$ to $k . x_{0}$. The minimal geodesic $c_{3}$ from $k . x_{0}$ to $g . x_{0}$ has lenght $d_{k^{-1} \cdot g}<t_{1}+d\left(x, g \cdot x_{0}\right)=d_{g}$ since otherwise $k \cdot x_{0}=x_{0}$. By induction there exists a Dirichlet neighbors chain from $k \cdot x_{0}$ to $g \cdot x_{0}$. So we get a Dirichlet neighbors chain from $x_{0}$ via $k \cdot x_{0}$ to $g \cdot x_{0}$, as required. 


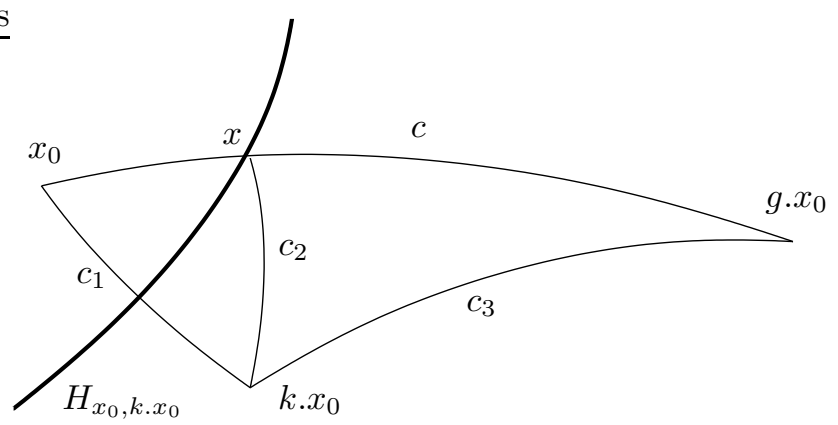

FIGURE 1.

Case 2. Suppose that $c$ meets $\bigcup_{e \neq k \in G} H_{x_{0}, k . x_{0}}$ for the first time at $x=c\left(\frac{1}{2} d_{g}\right) \in$ $H_{x_{0}, g . x_{0}}$ and that $x$ lies in no other central hypersurface. Then there exists an open convex ball $U$ with center $x$ which meets only $H_{x_{0}, g . x_{0}}$ from $\bigcup_{e \neq k \in G} H_{x_{0}, k . x_{0}}$. By lemma (2.2) the central hypersurface $H_{x_{0}, g . x_{0}}$ cuts $U$ in two connected components $\left\{y \in U: d\left(x_{0}, y\right) \gtrless d\left(g \cdot x_{0}, y\right)\right\}$ and is the boundary of both. One of them is in $D\left(x_{0}\right)^{o}$ and the other is in the interior $D\left(h . x_{0}\right)^{o}$ of a neighbor of $D\left(x_{0}\right)$. So $y=c\left(\frac{1}{2} d_{g}+\varepsilon\right) \in D\left(h . x_{0}\right)^{o}$ for some $\varepsilon>0$. Then $d\left(y, h . x_{0}\right)<d\left(y, x_{0}\right)$ and $d_{h^{-1} g}=d\left(h \cdot x_{0}, g \cdot x_{0}\right) \leq d\left(h \cdot x_{0}, y\right)+d\left(y, g \cdot x_{0}\right)<d_{g}$. By induction there is a Dirichlet neighbors chain from h. $x_{0}$ to $g . x_{0}$, thus also from $x_{0}$ to $g \cdot x_{0}$.

Case 3. Suppose that $c$ meets $\bigcup_{e \neq k \in G} H_{x_{0}, k . x_{0}}$ for the first time at $x=c\left(\frac{1}{2} d_{g}\right) \in$ $H_{x_{0}, g \cdot x_{0}} \cap H_{x_{0}, k \cdot x_{0}}$ for $k \neq g$. We have $d\left(x, x_{0}\right)=d\left(x, k \cdot x_{0}\right)$. Consider the minimal geodesic $c_{1}$ from $x_{0}$ to $k \cdot x_{0}$ which meets $H_{x_{0}, k \cdot x_{0}}$ in $c_{1}\left(\frac{1}{2} d_{k}\right)$. Then $d_{k}=d\left(x_{0}, k \cdot x_{0}\right)<d\left(x_{0}, x\right)+d\left(x, k \cdot x_{0}\right)$ since otherwise the curve following $c$ from $x_{0}$ to $x$ and then the minimal geodesic from $x$ to $k . x_{0}$ would be a minimal geodesic and could not have an angle $\neq 0$ at $x$ which implies that $k \cdot x_{0}=g \cdot x_{0}$. By induction there is Dirichlet neighbors chain from $x_{0}$ to $k \cdot x_{0}$. Moreover, $d_{k^{-1} g}=$ $d\left(k \cdot x_{0}, g \cdot x_{0}\right)<d\left(k \cdot x_{0}, x\right)+d\left(x, g \cdot x_{0}\right)=d_{g}$ since otherwise the piecewise minimal geodesic from $k \cdot x_{0}$ via $x$ to $g . x_{0}$ would be a minimal geodesic and thus $k \cdot x_{0}=x_{0}$. By induction again there is a Dirichlet neighbor chain from $k \cdot x_{0}$ to $g \cdot x_{0}$ which together with the first chain gives a chain from $x_{0}$ to $g . x_{0}$, as required.

2.6. Reflections. Let $(M, \gamma)$ be a connected complete Riemannian manifold. A reflection in $M$ is an isometry $s \in \operatorname{Isom}(M)$ such that for some fixed point $x_{0}$ of $s$ the tangent mapping $T_{x_{0}} s$ is a reflection in the Euclidean space $\left(T_{x_{0}} M, \gamma_{x_{0}}\right)$, with repect to a hyperplane: For some vector $0 \neq X_{x_{0}} \in T_{x_{0}} M$ we have $T_{x_{0}} s \cdot X_{x_{0}}=-X_{x_{0}}$, whereas $T_{x_{0}} s \mid X_{x_{0}}^{\perp}=$ Id.

Lemma. Let $s$ be a reflection on a complete connected Riemannian manifold $M$. Then we have:

(1) Every connected component $N$ of the fixed point set $M^{s}$ is a totally geodesic submanifold, and for each $x \in N$ the tangent mapping $T_{x} s$ equals the identity on $T_{x} N$ and $-\mathrm{Id}$ on $T_{x} N^{\perp}$.

(2) Every connected component $N$ of $M^{s}$ determines s completely as follows: For $y \in M$ there exists $x \in N$ such that $d(y, x)=\operatorname{dist}(y, N)$. Let $t \mapsto$ $\exp \left(t . Y_{x}\right)$ be a minimal geodesic which reaches $y$ at $t=1$. Then $s(y)=$ $\exp \left(-Y_{x}\right)$. 
(3) At least one connected component of $M^{s}$ is of codimension 1. Any such component is called a reflection hypersurface for $s$.

(4) For any $y \in M \backslash M^{s}$ we have $M^{s} \subseteq H_{y, s . y}$.

Proof. (1) Let $x_{0} \in M^{s}$ be a point such that $T_{x_{0}} s$ is a Euclidean reflection. Then $T_{x_{0}} s \circ T_{x_{0}} s=\operatorname{Id}_{T_{x_{0}} M}$, thus $s$ is also an involution. Consequently $T_{x} s$ is an Euclidean involution for each fixed point $x$, thus it is diagonalizable with eigenvalues +1 on the eigenspace $T_{x} N$ and eigenvalue -1 on the eigenspace $T_{x} N^{\perp}$ where $N$ is the connected component $N$ of $M^{s}$ containing $x$.

(2) Note that $Y_{x} \in T_{x} N^{\perp}$ and that $s\left(\exp \left(t . Y_{x}\right)\right)=\exp \left(t . T_{x} s . Y_{x}\right)=\exp \left(-t . Y_{x}\right)$.

(3) The connected component of $M^{s}$ containing $x_{0}$ is of codimension 1.

(4) For $x \in M^{s}$ let $c$ be a minimal geodesic from $x$ to $y$. Then $s \circ c$ is a minimal geodesic from $s . x=x$ to $s . y$. Thus $d(x, y)=d(x, s . y)$.

An example of a reflection $s$ which is generated by two different reflection hypersurfaces $H, H^{\prime}$ : Let $M=S^{1}, H=\{1\}, H^{\prime}=\{-1\}$, and let $s$ be complex conjugation. Another 2-dimensional example with three reflecting hypersurfaces is drawn in fig. 2. The 2-dimensional example in fig. 2 also shows that two different
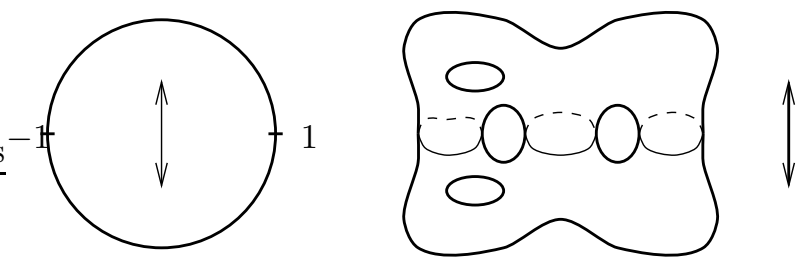

FIGURE 2. Reflections generated by different reflection hypersurfaces.

reflection hypersurfaces $H, H^{\prime}$ for the same reflection $s$ need not be parallel, i.e., $\operatorname{dist}\left(x, H^{\prime}\right)$ is not constant in $x \in H$.

In [30] one finds the following theorem: If an irreducible Riemannian symmetric space $M$ of noncompact type admits an involutory isometry whose fixed point set has codimension one, then $M$ is a real hyperbolic space. This extends a result of Iwahori [20] concerning irreducible Riemannian symmetric spaces of compact type; however, the proofs of these two results are substantially different.

2.7. Disecting reflections. An isometry $s \in \operatorname{Isom}(M)$ is called disecting if the complement of the fixed point set $M^{s}$ is not connected.

\section{Lemma.}

(1) A disecting isometry $s$ is a reflection.

(2) For a disecting reflection s the fixed point set $M^{s}$ disects $M$ into exactly 2 pieces. The reflection s permutes these two pieces.

(3) For a disecting reflection $s$ the fixed point set $M^{s}$ is a disjoint union of codimension 1 submanifolds.

(4) For a disecting reflection $s$ and any $y \in M \backslash M^{s}$ we have $M^{s}=H_{y, s . y}$.

Proof. (1) Since $M \backslash M^{s}$ is not empty and disconnected, the fixed point set $M^{s}$ which is a disjoint union of closed totally geodesic submanifolds contains at least one connected component of codimesion 1 . For any $x$ in a codimension 1 component 
$H$ the tangent mapping $T_{x} s$ equals $\operatorname{Id}_{T_{x} H}$ on $T_{x} H$ and is a nontrivial isometry on the 1-dimensional subspace $T_{x} H^{\perp}$, thus equals multiplication by -1 there.

(2) Let $x_{0} \in M \backslash M^{s}$. By (2.6.4) we have $M^{s} \subseteq H_{x_{0}, s . x_{0}}$. By (2.3.2) for the group $\{\mathrm{Id}, s\}$, removing the set $H_{x_{0}, s . x_{0}}$ decomposes $M$ into exactly two connected pieces. Thus the subset $M^{s} \subseteq H_{x_{0}, s . x_{0}}$ cannot decompose it into more than two pieces.

(3) The union $M_{1}^{s}$ of all codimension 1 connected components of $M^{s}$ also disects $M$ into two connected components, since removing also the components of higher codimension does not change connectedness any more. Let $N$ be a connected component of codimension $\geq 2$ of $M^{s}$. Then $N$ is contained in one component of $M \backslash M_{1}^{s}$ and $s$ thus has to map it into the other component, by (2). Thus $N$ is empty.

(4) By (2.6.4) we have $M^{s} \subseteq H_{y, s . y}$. Let $z \in H_{y, s . y}$. If $z \notin M^{s}$ then $z$ and $s(z)$ lie in different components of $M \backslash M^{s}$. Let $c_{1}$ be a minimal geodesic from $y$ to $z$, and let $c_{2}$ be a minimal geodesic (of the same length) from $s(y)$ to $z$. Then the broken geodesic $c_{1} c_{2}^{-1}$ from $y$ to $s(y)$ has to meet $M^{s}$ in some point $x \in M^{s}$ since $y$ and $s(y)$ lie in different components of $M \backslash M^{s}$. If $x$ is an inner point on $c_{2}$, say, then the broken geodesic following $s\left(c_{2}\right)$ from $y$ to $s(x)=x$ and then $c_{2}$ from $x$ to $z$ has the same length as $c_{2}$ and hence $c_{1}$. It has an angle at $x$ (otherwise $z=s(z)$ and we are done), thus there is a geodesic from $y$ to $z$ shorter than $c_{1}$, a contradiction.

2.8. Theorem. Let $M$ be a simply connected complete Riemannian manifold.

Then any reflection $\sigma$ on $M$ is disecting, and its fixed point set $M^{\sigma}$ is a connected orientable totally geodesic closed hypersurface.

Proof. Let $x \in M \backslash M^{\sigma}$ and let $H$ be a connected component of $M^{\sigma}$ of codimension 1 . Choose a minimal geodesic $c^{+}$from $x$ to $H$. It hits $H$ orthogonally by minimality, and thus we may continue it by $c^{-}=\sigma \cdot c^{+}$to obtain a geodesic $c_{0}$ from $x$ to $\sigma(x)$ which hits $H$ in exactly one point.

Suppose that $M \backslash H$ is connected. Then there exists a smooth curve $c_{1}$ from $x$ to $\sigma(x)$ in $M \backslash H$. Since $M$ is simply connected, there exists a smooth homotopy $h:[0,1] \times[0,1] \rightarrow M$ with $h(0, s)=x, h(1, s)=\sigma \cdot x, h(t, 0)=c_{0}(t)$, and $h(t, 1)=$ $c_{1}(t)$. We can also assume that $h$ is transversal to $H$. But then $h^{-1}(H)$ is a closed 1-dimensional submanifold in $[0,1]^{2}$ which hits the boundary exactly once in $[0,1] \times\{0\}$ and never in $\{0\} \times[0,1]$ or $\{1\} \times[0,1]$. So the connected component hitting once must hit again in $[0,1] \times\{1\}$. Thus $c_{1}$ hits $H$, a contradiction.

Thus $M \backslash H$ is not connected, and $H$ cuts $M$ into two components, $M^{+}$and $M^{-}$. Moreover, $M^{\sigma}=H$ since $\sigma$ interchanges $M^{+}$and $M^{-}$.

2.9. Reflection groups and chambers. Let $G \subset \operatorname{Isom}(M)$ be a discrete subgroup of isometries of a connected complete Riemannian manifold $M$ which is generated by all reflections contained in $G$. We shall call any such group $G$ a reflection group of $M$. By a (Weyl) chamber we mean the closure in $M$ of a connected component of the (open) complement of the union of all reflection hypersurfaces of all reflections in $G$. By an open (Weyl) chamber we mean the open interior $C^{o}$ of a Weyl chamber $C$. For a chamber $C$ a wall is a connected component of $C \cap M^{s}$ for a reflection $s$ if it contains a non-empty open subset of $M^{s}$ of codimension 1 in $M$. Two walls $F_{i}, F_{j}$ are called neighbours is the intersection $F_{i} \cap F_{j}$ has a connected component of codimension 2 . 
Lemma. For a chamber $C$ and any regular point $x_{0} \in C^{o}$ the Dirichlet domain $D\left(x_{0}\right)$ is contained in $C$. Thus $C$ is a union of Dirichlet domains of the form $D\left(g \cdot x_{0}\right)$, for all $g \in N_{G}(C)$.

Moreover, $G$ acts transitively on the set of all chambers.

Proof. Since the set $M_{\text {reg }}$ of regular points is open and dense in $M$, we may choose a regular $x_{0} \in C$. We claim that $C \supseteq D\left(x_{0}\right)$.

For $x \in M$ consider a minimal geodesic $c$ from $x_{0}=c(0)$ to $x=c(1)$. If $c$ hits a reflection hypersurface $H$ in $c(t)$ for $t<1$, we may consider the minimal geodesic from $x_{0}$ to $c(t)$, followed by the minimal geodesic from $c(t)$ to $s_{H}(x)$, which is a broken geodesic from $x_{0}$ to $s_{H}(x)$ of length $d\left(x_{0}, c(t)\right)+d\left(c(t), s_{H}(x)\right)=d\left(x_{0}, c(t)\right)+$ $d(c(t), x)=d\left(x_{0}, x\right)$. Since it has a proper angle at $c(t)$, a minimal geodesic from $x_{0}$ to $s_{H}(x)$ has length $d\left(x_{0}, s_{H}(x)\right)<d\left(x_{0}, x\right)$. Thus we see: Whenever the minimal geodesic from $x_{0}$ to a point $x$ hits a reflection hypersurface $H$ in an intermediate point, $d\left(x_{0}, s_{H}(x)\right)<d\left(x_{0}, x\right)$.

Now let $y \in D\left(x_{0}\right)$. Then $d\left(x_{0}, y\right)=\operatorname{dist}\left(x_{0}, G . y\right)$. By the statement above, any minimal geodesic from $x_{0}$ to $y$ can hit a reflection hypersurface at most in $y$. Thus $y \in C$.

That $G$ acts transitively on the set of all chambers follows from (2.3).

2.10. Examples of non-disecting reflections. We consider the real projective plane $\mathbb{R} \mathbb{P}^{2}$, with the metric induced from $S^{2}$, and a reflection $s=s_{H}$ on one line $H$ in it. Look at fig. 3 where the line at infinity $L$ is chosen orthogonal to $H$ so that $L$ is invariant under $s$. On the line $L$ one still has to identify antipodically. The fixed point set of $s$ consists of $H$ and the single point $x$ on $L$ farthest from $H$. There is only one chamber $C=\mathbb{R P}^{2} \backslash(H \cup\{x\})$ which is a punctured disk and is dense in $\mathbb{R P}^{2}$. But there are two Dirichlet domains $D(z)$ and $D(s z)$ depending on $z$ which for $z$ on the line through $x$ othogonal to $L$ meet in $H \cup L$. As another

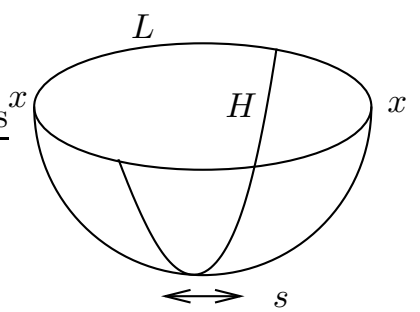

Figure 3. A reflection on $\mathbb{R} P^{2}$.

example, consider $M=S O(3)=\mathbb{R} P^{3}$ with the biinvariant metric. Then $g \mapsto g^{-1}$ is a non-disecting reflection whose fixed point set is the disjoint union of $\{e\}$ and some $\mathbb{R} P^{2}$. This reflection generates a Coxeter group.

2.11. Theorem. Let $M$ be a complete Riemannian manifold and let $G \subseteq \operatorname{Isom}(M)$ be a discrete group of isometries which is generated by all its reflections. Let $C$ be a Weyl chamber in $M$ for $G$. Let $F_{1}, F_{2}, \ldots$ be the walls of $C$ and let $s_{i}$ be the reflection with respect to the wall $F_{i}$.

Then the reflections $s_{1}, s_{2}, \ldots$ generate $G$, and they satisfy the following relations:

(1) $\left(s_{i}\right)^{2}=1$ 
(2) If two walls $F_{i}, F_{j}$ are neighbors then $\left(s_{i} s_{j}\right)^{n_{i j}}=1$ for some natural number $n_{i j}$.

See fig. 4 for a Weyl chamber in the Poincaré upper halfplane with infinitely many walls.

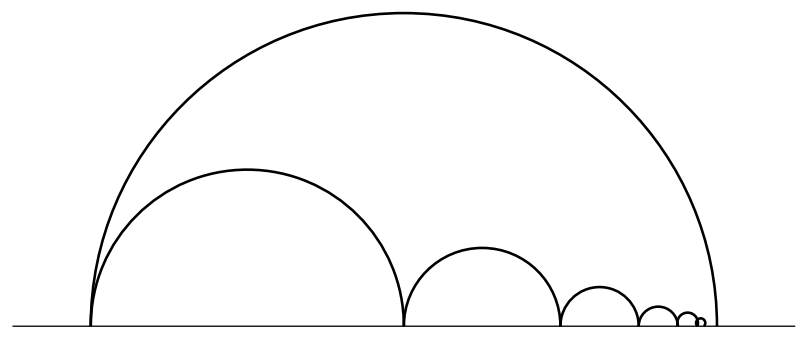

FIGURE 4.

A Weyl chamber in the Poincaré upper halfplane with infinitely many walls.

Proof. We prove that the reflections $s_{1}, s_{2}, \ldots$ generate $G$. Let $C^{\prime}$ be any other Weyl chamber in $M$. Then we choose a smooth curve $c:[0,1] \rightarrow M$ from a regular point $x_{0} \in C$ to a regular point $x^{\prime} \in C^{\prime}$ which changes Weyl chambers only transversally through open interiors of walls. First the curve passes from $C$ through the interior of a wall $F_{i_{1}}$ to a neighbor $s_{i_{1}}(C)$, and then through a wall $F$ of this chamber to the next. For the reflection $s_{F}$ in $F$ we have $s_{F}=s_{i_{1}} \cdot s_{i_{2}} \cdot s_{i_{1}}$ for some wall $F_{i_{2}}$ of $C$. If we now follow the curve $c$ through all interiors of walls we see that $C^{\prime}$ is of the form $C^{\prime}=g(C)$ for $g$ in the subgroup generated by $s_{1}, \ldots, s_{l}$. Any reflection in $G$ is of the form $s_{F}$ for some wall of some chamber $C^{\prime}$. But then the argument above shows that $s_{F}=g \cdot s_{i_{k}} \cdot g^{-1}$, so $G$ is generated by $s_{1}, s_{2}, \ldots$ as claimed.

Relations (1) and (2) follow, since if $x$ is an interior point of the face $f=$ $F_{i} \cap F_{j}$ (i.e., there are no other walls through $x$ ) then the stabilizer $G_{x}$ is faithfully and orthogonally represented in the two-dimensional space $T_{x}(f)^{\perp}$, and any finite subgroup of $O(2)$ which contains a reflection is a dihedral group.

2.12. Remark. In the setting of theorem (2.11) there might be more relations than specified in (2.11.1) and (2.11.2), see fig. 5. The left part of fig. 5 is a flat 2-
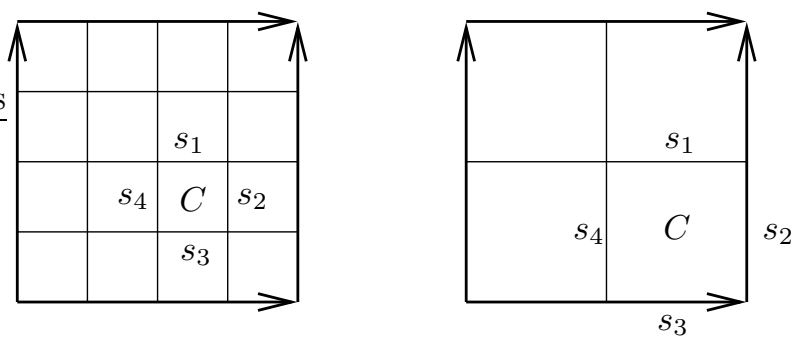

FiguRE 5. 2-tori with $\mathbb{Z}_{2}^{4}$ and $\mathbb{Z}_{2}^{2}$ as reflection groups.

torus with a chamber $C$ specified, with reflections $s_{1}, s_{2}, s_{3}, s_{4}$ and angular relations 
$\left(s_{i} s_{i+1}\right)^{2}=1$ for $i=1, \ldots, 4 \bmod (4)$ as decribed in (2.11.2). But moreover the relations $\left(s_{2} s_{4}\right)^{2}=1$ and $\left(s_{1} s_{3}\right)^{2}=1$ hold which are not described by (2.11.2).

In right hand part of fig. 5 we even have $s_{1}=s_{3}$ and $s_{2}=s_{4}$.

2.13. Lifting reflection groups to the universal covering. Let $\pi: \tilde{M} \rightarrow M$ be the universal covering of a Riemannian manifold $M$ with a reflection group $G$, and let $\pi_{1}(M)=\Gamma \subset \operatorname{Isom}(\tilde{M})$ be the group of deck transformations of $\pi$. Any isometry of $M$ can be lifted to an isometry of $\tilde{M}$. A lift $\tilde{s}$ of a reflection $s$ in $G$ is a reflection on $\tilde{M}$ if and only if it has a fixed point $\tilde{x} \in \tilde{M}$ with $\pi(\tilde{x})$ in a reflection hypersurface of $s$ in $M$. The group $\tilde{G}$ generated by all reflections which are lifts of reflections in $G$, is a reflection group in $\tilde{M}$ which is normalized by $\Gamma$ in $\operatorname{Isom}(\tilde{M})$. Then $\tilde{G} \Gamma$ is the group of all lifts of transformations in $G$, and $G=(\tilde{G} \Gamma) / \Gamma=\tilde{G} /(\tilde{G} \cap \Gamma)$. If $\tilde{C}$ is a chamber for $\tilde{G}$ in $\tilde{M}$ then $\pi(\tilde{C})$ is a chamber for $G$ in $M$, since the union of all reflections hypersurfaces of $\tilde{G}$ equals the inverse image under $\pi$ of the union of all reflection hypersurfaces of $G$.

Let $s$ be a reflection in $G$, and let $\tilde{s}$ be a reflection covering $s$ in $\tilde{G}$. According to (2.8) each reflection $\tilde{s}$ in $\tilde{G}$ is disecting, $\tilde{M}^{\tilde{s}}$ is one reflection hypersurface, and $\tilde{M} \backslash \tilde{M}^{\tilde{s}}$ consists of exactly two connected components $\tilde{M}_{+}^{\tilde{s}}$ and $\tilde{M}_{-}^{\tilde{s}}$.

If $G$ is generated by disecting reflections then $G$ acts simply transitively on the set of all chambers, see (3.5) below. The converse is not true, even if $G$ is a Coxeter group, see fig. 7 in (2.15).

Suppose that one (equivalently any) chamber is simply connected. Then $G$ acts simply transitively on the set of all chambers if and only if $\Gamma \subseteq \tilde{G}$. To see this, note that the universal cover $\pi: \tilde{M} \rightarrow M$ restricts to a diffeomorphism for each chamber $\tilde{C}$ in $\tilde{M}$ onto a chamber $C=\pi(\tilde{C})$ in $M$. If $\Gamma$ contains a nontrivial deck transformation $\gamma$, then for a chamber $\tilde{C}$ covering $C$ the set $\gamma(\tilde{C})$ is another chamber covering $C$. By $(2.10)$ and (3.5) there exists a unique $\tilde{g} \in \tilde{G}$ with $\tilde{g}(\tilde{C})=\gamma(\tilde{C})$. But then $\tilde{g}=\gamma$ if and only if $\tilde{g}$ covers $\operatorname{Id}_{M}$ in $G$.

2.14. Proposition. Let $G$ be a reflection group on a simply connected complete Riemannian manifold $M$. Then each chamber $C$ is simply connected.

Proof. Suppose for contradiction that some chamber $C$ is not simply connected: Let $c:[0,1] \rightarrow C$ be a closed smooth curve through a regular point $x_{0} \in C$ which is not contractible to the constant curve through $x_{0}$ in $C$ with fixed ends at $x_{0}$.

Since $M$ is simply connected there exists a smooth homotopy $h:[0,1] \times[0,1] \rightarrow$ $M$ with $h(0, t)=c(t), h(s, 0)=x_{0}, h(s, 1)=x_{0}$, and $h(1, t)=x_{0}$. We may assume that $h$ is transversal to each reflection hypersurface and to each intersection of such hypersurfaces, since these form a locally finite family by the discreteness of $G$. Thus for each intersection hypersurface $H_{i}$ the set $h^{-1}\left(H_{i}\right)$ is a 1 dimensional embedded submanifold of $[0,1]^{2}$ which does not meet the boundary, so it is a disjoint set of embedded circles in $C$ which may touch only the bottom boundary $\{0\} \times[0,1]$. Moreover, the sets $h^{-1}\left(H_{i}\right)$ are all pairwise transversal 1-dimensional submanifolds in $(0,1)^{2}$, or empty, since this is the case for the (geodesically closed) $H_{i}$ in $M$. Fig. 6 is an illustration. See [25], section 6, for transversality theorems on manifolds with corners. Now $h_{0}=c$ is completely contained in $\bar{c}$ and we consider the curve $h_{s}=h \mid(\{s\} \times[0,1])$ for $s$ moving from 0 to 1 . So we move $\{s\} \times[0,1]$ upwards inside $[0,1]^{2}$. If this line hits $h^{-1}\left(H_{i}\right)$ we start reflecting back into $C$ the point $h_{s}(t)$ for those $t$ which lie inside $h^{-1}\left(H_{i}\right)$. If we meet another $h^{-1}\left(H_{j}\right)$ we add the reflection $s_{H_{j}}$ at the right, etc. Since the different $h^{-1}\left(H_{i}\right)$ are transversal to each other 


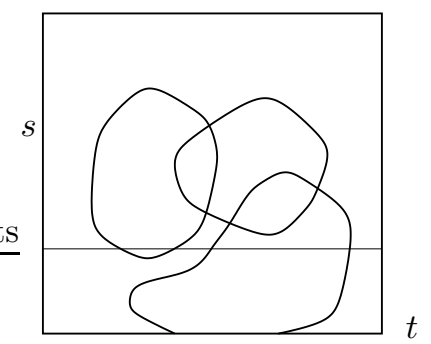

Figure 6 . The sets $h^{-1}\left(H_{i}\right)$ in $[0,1]^{2}$.

this is welldefined, in particular at $s=0$, where the sets $(\{0\} \times[0,1]) \cap h^{-1}\left(H_{i}\right)$ are disjoint by transversality. This proceedure transforms the smooth homotopy $h:[0,1]^{2} \rightarrow M$ to a continuous homotopy $\bar{h}:[0,1]^{2} \rightarrow C$ which contracts $c$ to $x_{0}$. Thus $C$ is simply connected which contradicts our assumption.

2.15. Maximal torus of a compact Lie group as manifold with reflections. Let $G$ be a semisimple compact Lie group with Lie algebra $\mathfrak{g}_{0}$ and let $T$ be a maximal torus in $G$. The Lie subalgebra $\mathfrak{t}_{0}$ to $T$ is then a Cartan subalgebra. Let $\Delta \subset \mathfrak{t}^{*}$ be the set of roots where $\mathfrak{t}=\mathfrak{t}_{0} \otimes \mathbb{C}$ is the complexification of $\mathfrak{t}_{0}$ and where $\mathfrak{t}^{*}$ is the dual space of $\mathfrak{t}$. Each root is purely imaginary on $\mathfrak{t}_{0}$. We have the following inclusion of lattices in $t^{*}$ :

$$
\mathbb{Z} \Delta \subseteq \Lambda_{\text {anal }} \subseteq \Lambda_{\text {alg }}, \quad \text { where }
$$

$\mathbb{Z} \Delta$ is the root lattice, generated by $\Delta$,

$\Lambda_{\text {anal }}$ is the lattice of analytically integral forms $\lambda \in L\left(\mathfrak{t}_{0}, i \mathbb{R}\right)$; they are characterized by the following property: whenever $H \in \mathfrak{t}_{0}$ satisfies $\exp (H)=1$ then $\lambda(H) \in 2 \pi i \mathbb{Z}$; equivalently: there exists a multiplicative character $\xi_{\lambda}: T \rightarrow S^{1}$ such that $e^{\lambda(H)}=\xi_{\lambda}(\exp (H))$ for all $H \in \mathfrak{t}_{0}$.

$\Lambda_{\text {alg }}$ is the weight lattice consisting of all $\lambda \in L\left(\mathfrak{t}_{0}, i \mathbb{R}\right)$ such that $2\langle\alpha, \lambda\rangle /|\alpha|^{2} \in \mathbb{Z}$ for all roots $\alpha \in \Delta$.

Now exp : $\mathfrak{t}_{0} \rightarrow T$ induces an isomorphism $\mathfrak{t}_{0} / \Lambda_{\text {anal }}^{*}=T$, where $\Lambda_{\text {anal }}^{*}$ is the dual lattice $\left\{X \in \mathfrak{t}_{0}: \lambda(X) \in \mathbb{Z}\right.$ for all $\left.\lambda \in \Lambda_{\text {anal }}\right\}$. Recall that $G$ has trivial center if and only if $\Lambda_{\text {anal }}=\mathbb{Z} \Delta$, that $G$ is simply connected if and only if $\Lambda_{\text {anal }}=\Lambda_{\text {alg }}$, that in general $\Lambda_{\text {anal }} / \mathbb{Z} \Delta$ is the center of $G$, and that the order of $\Lambda_{\text {alg }} / \mathbb{Z} \Delta$ equals the determinant of the Cartan matrix of $\mathfrak{g}$. The reflections on $T$ are induced by the reflections in the Weyl group in $\mathfrak{t}_{0}$; to visualize it we consider the reflections hyperplanes and the lattice $\Lambda_{\text {anal }}^{*}$ which consists of vectors orthogonal to the reflection hyperplanes. Then we consider a standard fundamental domain of the additive action of $\Lambda_{\text {anal }}^{*}$. We see that for $A_{1}^{k}=S U(2)^{k}$ all reflections in $T$ are disecting, but that for semisimple nonabelian $G$ we always get nondisecting reflections.

See fig. 7 for an example: It shows for $A_{2}=S U(3)$ the Cartan algebra $\mathfrak{t}_{0}$ as the universal covering of $T$ with the reflection hyperplanes (bold) for $W \rtimes \Lambda_{\text {anal }}^{*}$, the lattice $\Lambda_{\text {anal }}^{*}$, and the fundamental domain (dashed). The reflections on $T$ are not disecting, and the reflection group acts freely on the set of chambers in $T$, which are numbered. 


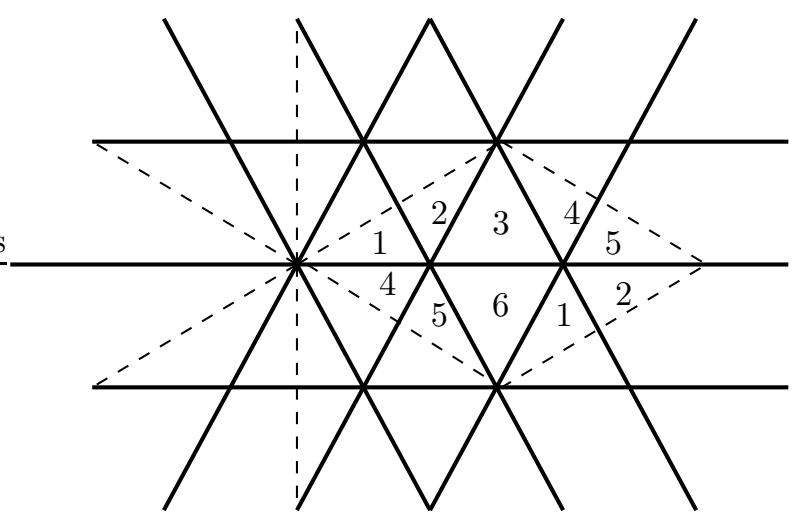

FIgURE 7. $\mathfrak{t}_{0}$ as universal covering of $T$ for $A_{2}=S U(3)$.

\section{Coxeter Riemannian manifolds}

3.1. Coxeter groups. [4] Recall that a Coxeter group is a group $G$ which is a quotient of a free group $G(S)$ with a set $S$ of generators by the subgroup generated by the relations $s^{2}=1$ and $\left(s s^{\prime}\right)^{n_{s, s^{\prime}}}=1$ for all $s, s^{\prime} \in S$, where $n_{s, s^{\prime}} \in\{1,2, \ldots, \infty\}$ indicates the order of $s s^{\prime}$ in $G$.

The set $S$ is called a set of standard generators of $G$, and $(G, S)$ is called a Coxeter system for $G$. Any subset $S^{\prime} \subset S$ generates a subgroup $G\left(S^{\prime}\right) \subset G$ such that $\left(G\left(S^{\prime}\right), S^{\prime}\right)$ is again a Coxeter system. $G\left(S^{\prime}\right)$ is called a Coxeter subgroup. The set of all Coxeter subgroups is a partially ordered set with respect to inclusion. A Coxeter system is described by a Coxeter diagram with vertices corresponding to the elements of $S$, where $s$ and $s^{\prime}$ are connected by $n_{s, s^{\prime}}-2$ edges if $\left(s s^{\prime}\right)^{n_{s s^{\prime}}}=1$ and $1<n_{s s^{\prime}}<\infty$. The Coxeter diagram of a Coxeter subgroup $\left(G\left(S^{\prime}\right), S^{\prime}\right)$ for $S^{\prime} \subset S$ is obtained from the Coxeter diagram of $(G, S)$ by deleting all vertices in $S \backslash S^{\prime}$ and all edges leading to such vertices.

The length $\ell(g)$ of an element $g \in G$ is the minimum number $l$ such that $g=$ $s_{i_{1}} \ldots s_{i_{l}}$ for $s_{i_{k}} \in S$. It satisfies $\ell\left(g g^{\prime}\right) \leq \ell(g)+\ell\left(g^{\prime}\right), \ell\left(g^{-1}\right)=\ell(g)$, and $\mid \ell\left(g^{\prime}\right)-$ $\ell(g) \mid \leq \ell\left(g^{\prime} g^{-1}\right)$.

In a Coxeter group $(G, S)$ let $P_{s}^{+}:=\{g \in G: \ell(s g)>\ell(g)\}$ and $P_{s}^{-}:=s P_{s}^{+}$. Then we have [4], iv, 1, 7:

(1) $\bigcap_{s \in S} P_{s}^{+}=\{e\}$.

(2) $G=P_{s}^{+} \sqcup P_{s}^{-}$(disjoint union) for each $s \in S$.

(3) Let $s, s^{\prime} \in S$ and $g \in G$. If $g \in P_{s}^{+}$and $g s^{\prime} \notin P_{s}^{+}$then $s=g s^{\prime} g^{-1}$.

Conversely, let $G$ be a group with a generating set $S$ of idempotents. Let $\left(P_{s}\right)_{s \in S}$ be a family of subset of $G$ which satisfies

(4) $e \in P_{s}$ for all $s \in S$.

(5) $P_{s} \cap s P_{s}=\emptyset$ for all $s \in S$.

(6) Let $s, s^{\prime} \in S$ and $g \in G$. If $g \in P_{s}$ and $g s^{\prime} \notin P_{s}$ then $s=g s^{\prime} g^{-1}$.

Then $(G, S)$ is a Coxeter system and $P_{s}=P_{s}^{+}$.

3.2. Riemannian Coxeter manifold. Let $G \subset \operatorname{Isom}(M)$ be a discrete subgroup of isometries of a complete Riemannian manifold $M$ which is generated by disecting reflections. Then $(M, G)$ is called a Riemannian Coxeter manifold. 
3.3. Coxeter manifolds of constant curvature. We recall some classical results.

Let $(G, S)$ be a Coxeter system such that $G$ is a finite group and let $S=$ $\left\{s_{1}, \ldots, s_{n}\right\}$. Then there exists a unique orthogonal representation of $G$ as a linear reflection group on an Euclidean space $\mathbb{R}^{n}$ such that the $s_{i}$ are reflections. The Weyl chamber associated to $S$ is a simplicial cone with walls $F_{1}, \ldots, F_{n}$ such that $s_{i}$ is the reflection in $F_{i}$. Then the angle $\alpha_{i j}$ between $F_{i}$ and $F_{j}$ is given by $\alpha_{i j}=\pi / n_{i j}$ where $\left(s_{i} s_{j}\right)^{n_{i j}}=1$ and $n_{i j}$ is minimal. In the following table we give the list of all finite Coxeter systems which are irreducible in the sense that they are not a direct product of two (commuting) Coxeter subsystems.

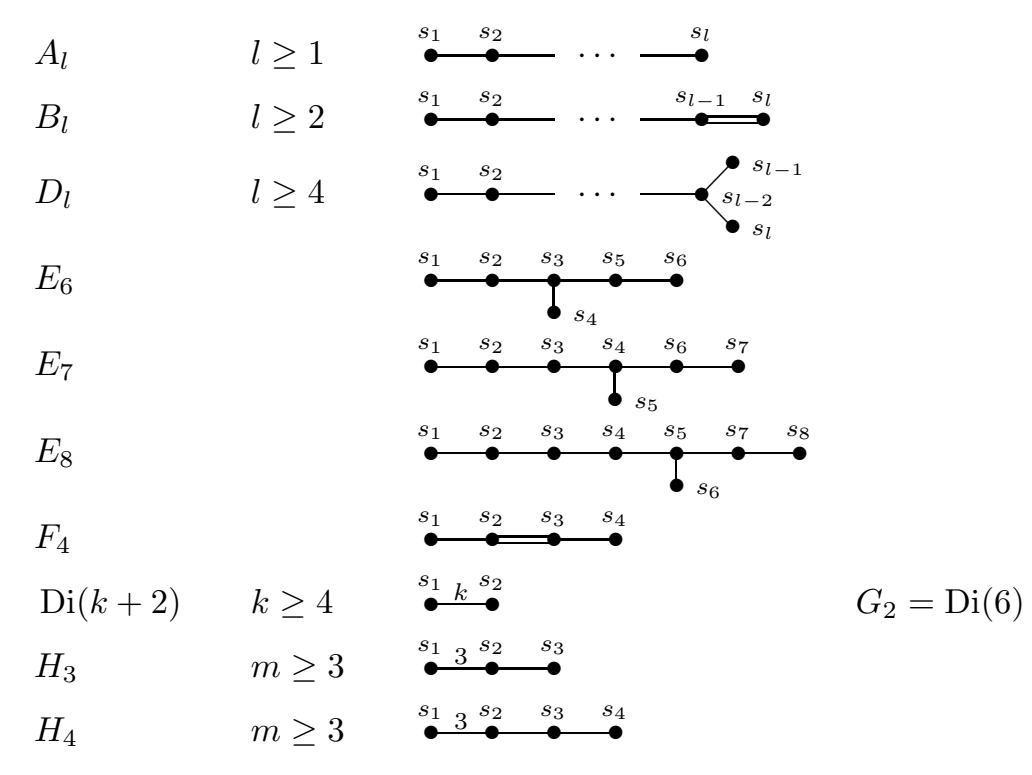

If the Coxeter group has no dihedral group $\operatorname{Di}(k+2)$ as direct factor, then the angle between two walls may only take the values $\alpha=\pi / n$ for $n=2,3,4,5,6$.

Conversely any simplicial cone with walls $F_{1}, \ldots, F_{n}$ having angles $\alpha_{i j}=\pi / n_{i j}$ between $F_{i}$ and $F_{j}$ where $n_{i, j} \in \mathbb{N}$, is the Weyl chamber of a uniquely given Coxeter system with finite Coxeter group, by [32], theorem 1 . The Coxeter diagram of $(G, S)$ contains also all information about the Weyl chamber. The angle between the walls $F_{i}$ and $F_{j}$ is $\alpha_{i j}=\pi / n_{i, j}$ where $n_{i j}-2$ is the number of edges connecting the vertices $s_{i}$ and $s_{j}$.

If $g \in G$ preserves a codimension $k$ face (an intersection of $k$ walls) $F=F_{i_{1}} \cap$ $\cdots \cap F_{i_{k}}$ which does not contain a line through 0 , then it it preserves it pointwise. Namely, $g$ has a fixed point $x$ in the interior of $F$ since $F$ is convex. By the lemma of Chevalley, $g$ is contained in the Coxeter subgroup generated by all reflections $s_{i}$ fixing $x$ which correspond to all walls through $x$. Since $x$ is an inner point of $F$, these walls also contain $F$. Thus $g$ fixes $F$ pointwise.

The angle in $F_{i_{1}} \cap \cdots \cap F_{i_{k}}$ between $F_{i_{1}} \cap \cdots \cap F_{i_{k}} \cap F_{i_{k+1}}$ and $F_{i_{1}} \cap \cdots \cap F_{i_{k}} \cap F_{i_{k+2}}$ is in general not of the form $\pi / n$; nevertheless it is uniquely determined by the Coxeter system. 
3.4. Example of Coxeter manifolds of non-constant curvature. Let $G$ be a linear reflection group on $\mathbb{R}^{n}$.

(1) Let $S$ be the unit sphere of $\mathbb{R}^{n}$. Then $G$ acts on $S$ and is generated by reflections. Choose a chamber $C$ in $\mathbb{R}^{n}$ and a $(n-1)$-ball $B$ in $C \cap S$. By surgery one may glue any compact $(n-1)$-dimensional manifold $M$ to $\partial B$ and do this in each chamber via the transformations of the group $G$. Obviously one can also put a $G$-invariant Riemannian metric on the resulting manifold, which then has complicated topology but carries a finite subgroup of the group of isometries which is generated by disecting reflections.

(2) Choose a chamber $C$ in $\mathbb{R}^{n}$ and within $C$ a regular point. Connect this point by a smooth curve to some point in each interior of each wall of $C$. Distributing this by $G$ into all chambers of $\mathbb{R}^{n}$ yields a graph on which $G$ acts. Now replace each point in the walls by a $S^{1}$ which lies completely in the interior of the wall, and replace the piece of the graph in the chamber $C$ by a smooth compact surface which all the $S^{1}$ 's as boundary components, meeting the walls orthogonally. Distribute this to all chambers by the $G$-action and obtain a smooth compact surface with induced Riemannian metric on which $G$ acts as a group of isometries generated by reflections.

3.5. Theorem. Let $(M, G)$ be a Riemannian Coxeter manifold. Then $G$ is a Coxeter group and $(G, S)$ is a Coxeter system for $G$, where $S$ is the set of reflections with respect to the walls of $C$. Moreover, $G$ acts simply transitively on the set of chambers.

Proof. We follow arguments from [12]. Let $Q$ be a chamber. For a reflection $s$ with respect to a wall $F$ of $Q$ we set

$$
P_{s}:=\left\{g \in G: g Q \subset M_{+}^{s}\right\}
$$

where $M_{+}^{s}$ is the connected componet of $M \backslash M^{s}$ which contains $Q$.

Lemma. $P_{s}=P_{s}^{+}=:\{g \in G: \ell(s g)>\ell(g)\}$.

Proof. It is sufficent to check the properties (3.1.4), (3.1.5), and (3.1.6). The first two properies are obvious. We check (3.1.6). Let $s, s^{\prime}$ be reflections with respect to walls $F, F^{\prime}$ of the chamber $Q$ and $g \in P_{s}$ but $g s^{\prime} \notin P_{s}$. The chambers $Q, s^{\prime} Q$ have a common wall $W$ and the chambers $g Q, g s^{\prime} Q$ have a common wall $g W$. Since they are on different sides of the hypersurface $M^{s}$, the wall $g W$ belongs to $M^{s}$, see Fig. 8. Then $s(g Q)=g s^{\prime} Q$ and $s^{\prime}, g^{-1} s g$ are two reflections which map

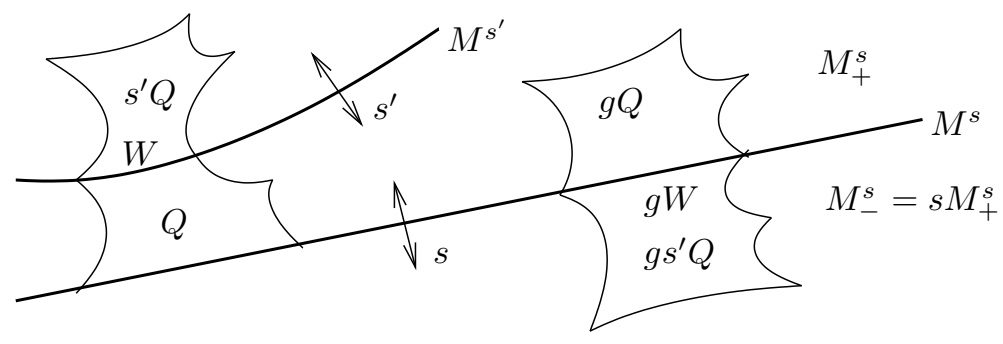

FiguRE 8.

$Q$ to $s^{\prime} Q$. Moreover $W \subset M^{s^{\prime}}$, and $g W \subset M^{s}$ implies $W \subset g^{-1} M^{s}=M^{g^{-1}} s g$, so that $M^{s^{\prime}}=M^{g^{-1}} s g$. Thus $s^{\prime}=g^{-1} s g . s g=g s^{\prime}$. 
Now the theorem follows from (3.1). Indeed, by (2.9) the group $G$ acts transitively on the set of chambers. Assume that $g Q=Q$ for some $g \in G$. Then $g \in \bigcap_{s \in S} P_{s}=\bigcap_{s \in S} P_{s_{i}}^{+}=1$ by property (3.1.1).

3.6. Corollary. A discrete group $G$ of isometries on a Riemannian manifold $M$ generated by reflections is a quotient of a Coxeter group.

Proof. This follows by (2.11), or by (2.13) and (3.5).

3.7. Question. Does there exist a discrete group of isometries which is generated by reflections but is not a Coxeter group? If so, can one characterize those which are Coxeter groups?

3.8. Corollary. Let $G$ be a reflection group on a complete connected Riemannian manifold $M$ such that $G$ acts freely and transitively on the set of all chambers, e.g., a Coxeter manifold. Let $C$ be a chamber. Then we have:

(1) $C$ is the Dirichlet domain associated with an interior point of $C$.

(2) Each chamber is convex and its interior consists of regular points.

(3) Any central hypersurface $H_{x_{0}, g . x_{0}}$ of a regular point $x_{0}$ and $1 \neq g \in G$ is a reflection hypersurface.

(4) $M_{r e g}=\bigcup_{g \in G} g C^{o}$.

(5) Let $F_{1}$ and $F_{2}$ be two walls of the chamber $C$ such that $F_{1}=g \cdot F_{2}$ for some $g \in G$. Then $F_{1}=F_{2}$.

(6) The natural projection $\pi: M \rightarrow M / G$ induces a homeomorphism $C \rightarrow$ $M / G$.

Proof. (1) By lemma (2.9) the chamber $C$ is a union of Dirichlet domains; but by (3.5) $G$ acts simply transitively on the set of chambers, thus $C$ is just one Dirichlet domain, by (2.3).

(2) By (1) and (2.3.2) each chamber consists of regular points. For convexity we have to show that any minimal geodesic arc between two points in $C$ is contained in $C$. This follows from [2], 3.5.

(3) By (1), the union of all open chambers equals the union of all open Dirichlet domains $D(x)$ for all regular points $x$. Thus also their complements in $M$ are the same: The union of all reflection hypersurfaces for $G$ in $M$ equals the union of all central hypersurfaces with respect to some (each) regular point. Thus the reflection hypersurfaces are exactly the central hypersurfaces $H_{g \cdot x_{0}, g^{\prime} \cdot x_{0}}$.

(4) If $x \in H_{x_{0}, g . x_{0}}$ then by (3) the isotropy group of $x$ is not trivial, so $x$ is not regular. Thus by $(2.3 .2)$ we have $M_{\text {reg }}=\bigcup_{g \in G} g \cdot C^{o}$.

(5) Let $F_{1}^{o}$ be the open interior of $F_{1}$ in some central hypersurface $H . F_{1}$ is contained in the intersection of exactly two chambers, namely $F_{1} \subseteq C \cap h . C$, where $h$ is the reflection in the hypersurface $H$. Also $F_{2}=g \cdot F_{1}=g \cdot C \cap g . h . C$, but one of the two chambers must be $C$. Thus $g=h^{-1}$ is the reflection at $H$ and so $F_{1}=F_{2}$.

(6) follows from (5) and from the fact that $G$ acts simply transitively on the set of all chambers.

3.9. Let $(M, G)$ be a connected Riemannian Coxeter manifold and let $C$ be a chamber. We denote by $W$ the set of walls of $C$ and by $G(W)$ the free group, generated by involutive generators $r_{F}$ corresponding to all walls $F \in W$. Since $G$ is generated by reflections with respect to walls in $W$, there is a natural homomorphism 
$G(W) \rightarrow G$. We denote its kernel by $R$. We define the normal subgroup $R_{a}$ of angular relations of $G(W)$ as follows:

Let $F_{i}, F_{j} \in W$ be neighboring walls with non empty intersection $f$ containing a codimension 2 submanifold, and let $F_{i}$ and $F_{j}$ have angle $\pi / n$ for a natural number $n$ along some codimension 2 connected component of $f$, then $\left(r_{F_{i}} r_{F_{j}}\right)^{n}$ is a generator of $R_{a}$ in $G(W)$.

We denote by $M_{i}, i=2,3$ the complement in $M$ of the union of codimension $\geq i$ intersections of reflection hypersurfaces. Note that these intersections are totally geodesic submanifolds as fixed point sets of finitely many isometries.

Theorem. In this situation, the group $R_{a}$ of angular relations is a normal subgroup of the group $R$ of all relations in $G$. Moreover, $\pi_{1}\left(M_{3}, x_{0}\right)=\pi_{1}\left(M, x_{0}\right)$ and $\pi_{1}\left(C^{o}, x_{0}\right)=\pi_{1}\left(C, x_{0}\right)$, and we have the following exact sequences of groups:

$$
\begin{gathered}
\{1\} \rightarrow \pi_{1}\left(C^{o}, x_{0}\right) *_{e} G(W) \rightarrow \pi_{1}\left(M_{2}, x_{0}\right) \rightarrow G(W) \rightarrow G \rightarrow\{1\} \\
\{1\} \rightarrow \pi_{1}\left(C, x_{0}\right) *_{e} G(W) / R_{a} \rightarrow \pi_{1}\left(M, x_{0}\right) \rightarrow G(W) / R_{a} \rightarrow G \rightarrow\{1\}
\end{gathered}
$$

where for groups $H$ and $G$ the group $H *_{e} G$ is the kernel of the projection $p_{G}$ : $H * G \rightarrow G$ from the free product to $G$. In particular,

$$
\begin{gathered}
\pi_{1}\left(M_{2}, x_{0}\right) /\left(\pi_{1}\left(C^{o}, x_{0}\right) *_{e} G(W)\right)=R, \\
\pi_{1}\left(M, x_{0}\right) /\left(\pi_{1}\left(C, x_{0}\right) *_{e} G(W) / R_{a}\right)=R / R_{a} .
\end{gathered}
$$

Proof. By (3.8.6) the composition $C \rightarrow M \rightarrow M / G$ is a homeomorphism thus $\pi_{1}\left(C, x_{0}\right) \rightarrow \pi_{1}\left(M, x_{0}\right)$ is injective. By restriction $C \cap M_{2} \rightarrow M_{2} \rightarrow M_{2} / G$ is also a homeomorphism thus $\pi_{1}\left(C \cap M_{2}, x_{0}\right) \rightarrow \pi_{1}\left(M_{2}, x_{0}\right)$ is injective. By (3.8.5) we have $\pi_{1}\left(C^{o}, x_{0}\right)=\pi_{1}\left(C, x_{0}\right)$ since a closed curve in $C$ may be deformed into $C^{o}$.

Any element in $\pi_{1}\left(M, x_{0}\right)$ can be represented by a closed smooth curve $c$ through $x_{0}$ in $M$ which we may assume to be transversal to all intersections of walls. By dimension, $c$ lies in $M_{2}$ and first meets a wall $F_{1}$ of $C$ transversally. Next it meets a wall $s_{F_{1}}\left(F_{2}\right)$ of $s_{F_{1}}(C)$ transversally. And so on until it comes back to $x_{0}$. We assign to $c$ the expression (word) $r_{F_{1}} r_{F_{2}} \ldots r_{F_{k}}$ in $G(W)$. A homotopy moving $c$ in $M_{2}$ just allows cancellations in this expression using $r_{F}^{2}=1$. Replacing the $r_{F}$ in this expression by the corresponding $s_{F}$ we get an element in the reflection group $G$ which maps $C$ to $C$ and thus is the identity, by theorem (3.5).

Let $f_{i}$ be a fixed curve from $x_{0}$ to $s_{F_{i}}\left(x_{0}\right) \in s_{F_{i}}(C)$ hitting $F_{i}$ once transversally. Any expression $r_{F_{1}} \ldots r_{F_{k}}$ in $G(W)$ which maps to the identity in $G$, is assigned to the closed curve in $M_{2}$ which first follows $f_{1}$ from $x_{0}$ to $s_{F_{1}}\left(x_{0}\right)$, then $s_{F_{1}} \circ f_{2}$ from $s_{F_{1}}\left(x_{0}\right)$ to $s_{F_{1}} s_{F_{2}}\left(x_{0}\right)$, etc., until it ends again in $x_{0}$. Thus the sequence is exact at $G(W)$.

A curve representing an element in $\pi_{1}\left(M_{2}, x_{0}\right)$ which is transversal to walls can be described, up to 'transversal' homotopy, by a word $c_{0} r_{F_{1}} c_{1} r_{F_{2}} c_{2} \ldots r_{F_{k}} c_{k}$ where:

- $c_{i} \in \pi_{1}\left(s_{F_{1}} s_{F_{2}} \ldots s_{F_{i}}\left(C^{o}\right), s_{F_{1}} s_{F_{2}} \ldots s_{F_{i}}\left(x_{0}\right)\right) \cong \pi_{1}\left(C^{o}, x_{0}\right)$,

- $r_{F_{i}}$ stands for the curve $s_{F_{1}} s_{F_{2}} \ldots s_{F_{i-1}}\left(f_{i}\right)$.

- $s_{F_{1}} s_{F_{2}} \ldots s_{F_{k}}=e$ in $G$ since the curve is closed.

Thus the word describes a unique element of the free product $\pi_{1}\left(C^{o}, x_{0}\right) * G(W)$ which is in the kernel of $\pi_{1}\left(C^{o}, x_{0}\right) * G(W) \rightarrow G$. The curve in $\pi_{1}\left(M_{2}, x_{0}\right)$ maps to $e \in G(W)$ if and only if the word above also satisfies

- $r_{F_{1}} r_{F_{2}} \ldots r_{F_{k}}=e$ in $G(W)$. 
These are the elements of $\pi_{1}\left(C^{o}, x_{0}\right) *_{e} G(W)$.

So the first sequence is left exact, and surjectivity at $G$ follows from (2.11).

The second exact sequence follows from the first one: any homotopy in $M$ between smooth curves in $M_{2}$ may be assumed to be transversal to all intersections of reflection hypersurfaces of codimension $\geq 2$. Then it avoids all intersection of codimension $\geq 3$, so it lies in $M_{3}$. Thus $\pi_{1}\left(M, x_{0}\right)=\pi_{1}\left(M_{3}, x_{0}\right)$. If the homotopy meets an intersection $f=F_{1} \cap F_{2}$ transversely, moving the curve through $f$ means a cancellation in the expression assigned to the curve which is given by the corresponding generator $\left(r_{F_{1}} r_{F_{2}}\right)^{n}$ of $R_{a}$.

3.10. Theorem. Let $(M, G)$ be a simply connected Riemannian Coxeter manifold and let $C$ be a chamber. Then we have:

(1) In terms of (3.9) we have $R_{a}=R$. In other words, the relations (2.11.1) and (2.11.2) generate all relations of the Coxeter system $(G, S)$.

(2) The stabilizer $G_{x}$ of a point $x \in C$ is a finite Coxeter group generated by reflections with respect to the walls $F_{i_{1}}, \ldots, F_{i_{k}}$ through $x$. Moreover, if $G_{x}$ has no factor isomorphic to the dihedral group $D(m)$ for $m=5$ or $>6$, then the angles between two walls through $x$ take values $\pi / n$ for $n=2,3,4,6$.

For linear Coxeter groups this result was proved by Vinberg [32].

Proof. (1) This follows from $\pi_{1}\left(M, x_{0}\right)=R / R_{a}$ from (3.9).

(2) Let $g=s_{F_{1}} \ldots s_{F_{j}} \in G_{x}$. Since any $h \in G$ preserves the union of all reflection hypersurfaces, $g$ permutes the set of reflection hypersurfaces through $x$. Thus $g(f)=f$ where $f$ is the connected component of $F_{i_{1}} \cap \cdots \cap F_{i_{k}}$ containing $x$. Then $C \cap g C \supseteq f$.

We shall use the method of proof of theorem (3.9). Now choose a regular point $x_{0} \in C$ near $x$ and a curve $c_{1}$ in $M_{2}$ from $x_{0}$ to $g x_{0}$ which transverses the walls $F_{j}$, then $s_{F_{1}}\left(F_{2}\right)$, etc. Choose a second smooth curve $c_{2}$ in $M_{2}$ from $x_{0}$ to $g x_{0}$ in $M_{2}$ which is near $x$ so that it intersects only walls through $x$. Then we choose a homotopy in $M$ between $c_{1}$ and $c_{2}$ which we may assume to be transversal to all codimension $\geq 2$ intersections of reflection hypersurfaces. Then it is in $M_{3}$ and cuts intersections of two reflection hypersurfaces transversely. Moving $c_{1}$ to $c_{2}$ via this homotopy amounts to do angular cancellations (in $R_{a}$ ) in the representation of $g$. Thus $g$ is represented also as a word in reflections in hypersurfaces through $x$ according to the transversing of $c_{2}$ of the corresponding walls.

\section{Riemannian manifolds With CORNERs of CoXeter type}

4.1. Manifolds with corners. For more details see [25], section 2. A quadrant $Q \subset \mathbb{R}^{n}$ of index $k$ is a subset of the form $Q=\left\{x \in \mathbb{R}^{n}: l_{1}(x) \geq 0, \ldots, l_{k}(x) \geq 0\right\}$ where $l_{1}, \ldots, l_{k}$ are independent linear functionals on $\mathbb{R}^{n}$. If $x \in Q$ and exactly $j$ of the $l_{i}$ vanish on $x$ then $x$ is called a corner of index $j$. For an open subset $U \subset Q$ a mapping $f: U \rightarrow \mathbb{R}^{p}$ is called $C^{r}(0 \leq r \leq \infty)$ if all partial derivatives of $f$ of order $\leq r$ exist and are continuous on $U$. By the Whitney extension theorem this is the case if and only if $f$ can be extended to a $C^{r}$ function $\tilde{f}: \tilde{U} \rightarrow \mathbb{R}^{p}$, where $\tilde{U} \subset \mathbb{R}^{n}$ is open and $U=\tilde{U} \cap Q$. If $f: U \rightarrow U^{\prime}$ is a diffeomorphism between open subsets of quadrants in $\mathbb{R}^{n}$ then the index of $x \in U$ equals the index of $f(x) \in U^{\prime}$.

A smooth manifold with corners $M$ is defined in the usual way: it is modelled on open subsets of quadrants in $\mathbb{R}^{n}$; a chart on $M$ is a diffeomorphism $u: U \rightarrow u(U)$ from an open subset $U \subset M$ onto an open subset $u(U)$ of a quadrant in $\mathbb{R}^{n}$, where 
$n=\operatorname{dim}(M)$. The chart $(U, u, Q)$ is called centered on $x$ if $u(x)=0$. A point $x \in M$ is called a corner of index $j$ if there is a chart $(U, u, Q)$ of $M$ with $x \in U$ and and $u(x)$ a corner of index $j$ in $Q$.

A subset $N \subset M$ is called a submanifold with corners of the manifold with corners $M$, if for any $y \in N$ there is a chart $(U, u, Q)$ of $M$ centered at $y$ and there is a quadrant $Q^{\prime} \subseteq \mathbb{R}^{k} \subseteq \mathbb{R}^{n}$ such that $Q^{\prime} \subseteq Q$ and $u(N \cap U)=u(U) \cap Q^{\prime}$. A submanifold with corners $N$ of $M$ is called neat if the index in $N$ of each $y \in N$ coincides with its index in $M$. Only neat submanfolds have tubular neigborhoods.

Let us denote by $\partial^{j} M$ the set of all corners of index $j$ of $M$. Note that $\partial^{0} M=M$. Then each $\partial^{j} M$ is a submanifold without boundary of $M$. Let $\partial M:=\bigcup_{j \geq 1} \partial^{j} M$.

Each closure (in $M$ ) of a connected component of $\partial^{j} M$ is a submanifold with cornes of $M$ which is called a codimension $j$ face of $M$; it is of dimension $n-j$. A codimension 1 face is also called a wall. A face is not neat. The set of all faces is a partially ordered set with respect to inclusion.

The tangent bundle of a manifold with corners $M$ is constructed in the following way: Let $\left(U_{\alpha}, u_{\alpha}, Q_{\alpha}\right)$ be an atlas of $M$. Then $T M$ is the quotient space of the disjoint union $\bigsqcup_{\alpha}\left(\{\alpha\} \times U_{\alpha} \times \mathbb{R}^{n}\right) / \sim$ by the following equivalence relation: $(\alpha, x, v) \sim(\beta, y, w)$ if $x=y$ and $d\left(u_{\beta} \circ u_{\alpha}^{-1}\right)\left(u_{\alpha}(x)\right) v=w$. Then $\pi_{M}: T M \rightarrow M$ is a smooth vector bundle, and the total space $T M$ is again a manifold with corners: the corners are all in the base.

A tangent vector $X$ is called inner (short for: not outer) if there is a smooth curve $c:[0,1) \rightarrow M$ with $\dot{c}(0)=X$. If $X \in T_{x} M$ and if $(U, u, Q)$ is a chart with $x \in U$, and if the quadrant $Q$ is given by the independent linear functionals $l_{1}, \ldots, l_{k}$, and if $T u(X)=(u(x), v) \in u(U) \times \mathbb{R}^{n}$, then $X$ is inner if and only if the following holds: If $l_{i}(u(x))=0$ then $l_{i}(v) \geq 0$, for all $i$. Let us call the tangent vector strictly inner if $l_{i}(u(x))=0$ implies $l_{i}(v)>0$, for all $i$. Let us denote the space of all inner vectors by ${ }^{i} T M \subset T M$. It is not a manifold with corners any more. For example, ${ }^{i} T[0, \infty)=\{(x, v): x \geq 0, x=0 \Longrightarrow v \geq 0\}$.

An inner vector field on $M$ is a smooth vector field $X: M \rightarrow T M$ whose values are all inner tangent vectors. By pasting local solutions one can show that there exists a smooth open semiflow of $X$ in the following sense: There is a set $W \subset \mathbb{R} \times M$ containing $\{0\} \times M$ and $\left[0, \varepsilon_{x}\right) \times\{x\}$ for some $\varepsilon_{x}>0$ for each $x \in M$ and a smooth mapping $\mathrm{Fl}^{X}: W \rightarrow M$ with $\mathrm{Fl}_{0}^{X}(x)=x$ and $\frac{d}{d t} \mathrm{Fl}_{t}^{X}(x)=X\left(\mathrm{Fl}_{t}^{X}(x)\right)$. But $\mathrm{Fl}_{t}^{X}$ is not even a local diffeomorphism (it may map a corner to an interior point).

By a partition of unity argument one can show that there exists a smooth vector field $Y$ on $M$ which is strictly inner, and one may adapt it in such a way that its flow $\mathrm{Fl}_{t}^{Y}$ is defined everywhere on $M$ for $0 \leq t \leq \varepsilon$ for $\varepsilon>0$. Then $\mathrm{Fl}_{\varepsilon}^{Y}$ maps $M$ into its interior $M \backslash \partial M$. Thus: Each manifold with corners $M$ is a submanifold with corners of a manifold without boundary of the same dimension. See also [18].

Let $X$ be a vector field on $M$ which is tangential to the boundary: if $x \in \partial^{j} M$ then $X(x) \in T_{x} \partial^{j} M$ for all $j$. Then there exists a local flow for $X$ for positive and for negative time; the set $W \subset \mathbb{R} \times M$ is open.

4.2. Equipment of a manifold with corners. Let $M$ be an $n$-dimensional manifold with corners. Consider a surjective mapping $s$ from the set $W$ of all walls (codimension 1 faces) of $M$ onto the set of generators $S$ of a Coxeter system $(G, S)$ (see (3.1)). Any face $f$ of $M$ of codimension $k$ is the intersection of $k$ many walls $W_{1}, \ldots W_{k}$ (but not conversely). Then we extend the map $s$ to a map $s$ from the 
set of faces of $M$ into the set of Coxeter subgroups of $G$ as follows :

$$
s: f=F_{1} \cap \ldots \cap F_{k} \mapsto s(f)=G\left(s\left(F_{1}\right), \ldots s\left(F_{k}\right)\right)
$$

where $G\left(s\left(F_{1}\right), \ldots s\left(F_{k}\right)\right.$ is the subgroup of $G$ generated by $s\left(F_{1}\right), \ldots s\left(F_{k}\right)$.

The mapping $s$ is called a Coxeter equipment of $M$ by the Coxeter system $(G, S)$, if $G(F)$ is a finite group for each face of codimension $\geq 1$. It follows that $s$ is an partial order reversing homomorphism of the poset of all faces of $M$ into the poset of all Coxeter subgroups of the Coxeter system $(G, S)$ if we also put $s(\emptyset)=G$. Note that $s(M)=\{1\}$.

4.3. Riemannian manifolds with corners. A Riemannian metric on a manifold with corners $M$ is as usual a smooth section $\gamma: M \rightarrow S_{+}^{2} T^{*} M$. So it can be smoothly extended to a Riemannian metric on a manifold without boundary of the same dimension which contains $M$ as a submanifold with corners. If the Riemannian metric has the property that each closure of a face is a totally geodesic submanifold, then for each each inner tangent vector $X_{x} \in{ }^{i} T_{x} M$ the geodesic $t \mapsto \exp _{x}\left(t X_{x}\right)$ is defined for small nonnegative $t$.

This can be expressed by the property of the geodesic spray to be 'inner' and 'tangential' to all boundary strata $\partial^{j} M$, see [25], section 2. In detail: A vector $\Xi \in T T M$ is called an inner tangent vector to ${ }^{i} T M$ if there exists a smooth curve $c:[0, \varepsilon) \rightarrow T M$ with $\pi_{T M}(\Xi)=c(0), c([0, \varepsilon)) \subset{ }^{i} T M$, and $c^{\prime}(0)=\Xi$. For example, let $Q=\left\{x \in \mathbb{R}^{n}: l_{1}(x) \geq 0, \ldots, l_{k}(x) \geq 0\right\}$ be a quadrant and let $(x, u) \in{ }^{i} T Q$. A vector $(x, u ; v, w) \in T^{2} Q$ then is inner to ${ }^{i} T M$ if and only if:

(1) If $x$ is inner, so $u$ is arbitrary, then $(v, w)$ is arbitrary.

(2) If $l_{i}(x)=0$ and $l_{i}(u)>0$ then $l_{i}(v) \geq 0$ and $w$ is arbitrary.

(3) If $l_{i}(x)=0$ and $l_{i}(u)=0$ then $l_{i}(v) \geq 0$ and $l_{i}(w) \geq 0$.

Let us denote by ${ }^{i} T^{2} M$ the set of all vectors which are inner to ${ }^{i} T M$. A spray $S$ on the manifold with corners $M$ is a smooth mapping $S: T M \rightarrow T^{2} M$ such that

(4) $T\left(\pi_{M}\right) \circ S=\operatorname{Id}_{T M}$.

(5) $\pi_{T M} \circ S=\operatorname{Id}_{T M}$.

(6) $T\left(m_{t}\right) \cdot S(X)=\frac{1}{t} S(t . X)$ for $0 \neq t \in \mathbb{R}$, where $m_{t}: T M \rightarrow T M$ is scalar multiplication by $t$.

The spray is called inner if $S\left({ }^{i} T M\right) \subset{ }^{i} T^{2} M$ and it is called tangential if moreover $S$ is tantent to each boundary stratum: $S\left(T \partial^{j} M\right) \subset T^{2}\left(\partial^{j} M\right)$.

If $\gamma$ is a smooth Riemannian metric on the manifold with corners $M$, then we may extend $\gamma$ to a Riemannian metric $\tilde{\gamma}$ on a suitable open manifold $\tilde{M}$ of the same dimension which contains $M$ as submanifold with boundary. We may compute the geodesic (Levi-Civita) spray $\tilde{S}$ of $\tilde{\gamma}$ and restrict it again to $T M$. This spray is an inner tangential spray if and only if in $(M, \gamma)$ all closures of faces are totally geodesic submanifolds, and we have exp $=\pi_{M} \circ \mathrm{Fl}_{1}^{S}$.

Thus we conclude (see also [25], 2.10):

Lemma. [25], 2.10 Let $\gamma$ be a Riemannian metric on a manifold with corners $M$ such that all faces are totally geodesic. Then there exists a suitable open neighborhood $V$ of the zero section in $T^{i} M$ such that the geodesic exponential mapping $\exp : V \rightarrow M$ is defined. If $V$ is small enough then $\exp$ has the following properties:

(1) $\exp \left(0_{x}\right)=x$ for all $x \in M$.

(2) $\exp _{x}: V_{x}:=V \cap T_{x}^{i} M \rightarrow M$ is a diffeomorphism of $V_{x}$ onto an open neighborhood $W_{x}$ of $x$ in $M$. 
(3) $V_{x}$ is the intersection of an open ball $B_{x} \subset\left(T_{x} M, \gamma_{x}\right)$ with a quadrant $Q_{x} \subset T_{x} M$.

(4) The mapping $\left(\pi_{M}, \exp \right): V \rightarrow M \times M$ is a diffeomorphism onto an open neighborhood of the diagonal in $M \times M$.

(5) exp restricts to the exponential mapping of the induced Riemannian metric on each closure of a face.

4.4. Riemannian chambers and their Coxeter equipment. An Riemannian chamber is a manifold with corners $C$ with a Riemannian metric $\gamma$ such that each face is totally geodesic and such that the following two conditions (1) and (2) are satisfied.

(1) The angle between neighboring walls $W_{i}$ and $W_{j}$ is a constant of the form $\pi / n_{i j}$ for $n_{i j} \in \mathbb{N}$ along any codimension 2 connected component of $W_{i} \cap W_{j}$.

Let $V \subset T^{i} C$ be small as in (4.3). Then $\exp _{x}: V_{x}=V \cap T_{x}^{i} C \rightarrow W_{x} \subset C$ is a diffeomorphism. Recall from (4.3) that $V_{x}$ is the intersection of an open ball $B_{x}$ in $\left(T_{x} C, \gamma_{x}\right)$ with a quadrant $Q_{x}$ whose walls contain the inverse images under $\exp _{x}$ of the closed walls of $C$ containing $x$. The angles between the hyperplanes $T_{x} W_{i}$ and $T_{x} W_{j}$ in the Euclidean space $\left(T_{x} C, \gamma_{x}\right)$ are exactly $\pi / n_{i j}$, by (1). By [32], theorem 1 , this equivalent to the fact that the group $G_{x} \subset O\left(T_{x} C, g_{x}\right)$ generated by the reflections in the hyperplanes $T_{x} W_{i}$ is a finite Coxeter group with fundamental Weyl chamber $\mathbb{R}_{\geq 0} \cdot V_{x}$.

Consider the pullback Riemannian metric $\left(\exp _{x} \mid V_{x}\right)^{*} \gamma$ on $V_{x}$. Now we can formulate the second condition:

(2) If we extend the Riemannian metric $\left(\exp _{x} \mid V_{x}\right)^{*} \gamma$ on $V_{x}$ to the ball $B_{x}=$ $G_{x} . V_{x}$ by using the elements of $G_{x}$ as isometries, then the resulting $G_{x^{-}}$ invariant Riemannian metric $\tilde{\gamma}_{B_{x}}$ on $B_{x}$ is smooth.

If $G$ is a discrete group of isometries of a complete Riemannian manifold $(M, \gamma)$ which is generated by disecting reflections, and if a chamber $C$ is also a Dirichlet domain, then obviously $(C, \gamma)$ is a Riemannian chamber.

Proposition. Any Riemannian chamber $C$ carries a universal Coxeter equipment.

Proof. Let $\left\{W_{i}\right\}$ be the set of all walls of $C$. For each wall $W_{i}$ of $C$ we take a generator $s_{i}$. Then let $G$ be the group generated by all $s_{i}$, with relations $\left(s_{i} s_{j}\right)^{n_{i j}}=1$, whenever $W_{i} \cap W_{j} \neq \emptyset$ and where the angle between $W_{i}$ and $W_{j}$ is $\pi / n_{i j}$. Then $G$ is a Coxeter group with Coxeter system $\left(G,\left\{s_{i}\right\}\right)$. For each $x \in C$ we constructed in (4.4) a linear Coxeter group $G_{x} \subset O\left(T_{x} C, g_{x}\right)$ which is generated by those $s_{i}$ for which $x \in W_{i}$. Obviously, $G_{x}$ is a finite subgroup of $G$. Moreover, let $F=W_{1} \cap \ldots W_{k}$ be a nonempty face. Then $G(F)$ is generated by the reflections $s_{1}, \ldots, s_{k}$ which satisfy pairwise $\left(s_{i} s_{j}\right)^{n_{i j}}=1$ for $2 \leq n_{i j}<\infty$. Thus $G(F)$ is finite for each nonempty face.

The Coxeter equipment constructed in this proposition is called universal since the mapping $s$ is injective. Other Coxeter equipments are possible, if different walls are mapped to the same generator in such a way, that the isotropy group of each face $F$ stay isomorphic to $G(F)$ as above, and the full group is still a Coxeter group.

Thus we say that a Coxeter equipment $s$ of the Riemannian chamber $C$ is admissible, if for any two different walls $W_{i}$ and $W_{j}$ with nonempty intersection the element $s\left(W_{i}\right) s\left(W_{j}\right)$ has order exactly $n_{i j}$ in $G$, where the angle between $W_{i}$ and 
$W_{j}$ is $\pi / n_{i j}$. The right hand side of fig. 2 gives an example of a not universal equipment.

4.5. The Coxeter Riemannian manifolds associated with a Riemannian chamber. Note that by (2.11) and (3.9) the Weyl chamber $C$ of a Coxeter $G$ manifold $M$ has the natural structure of a Riemannian chamber with the admissible equipment $s: F \mapsto s(F)=\left\langle s \in S: M^{s} \supset F\right\rangle$. In the non-disecting case this is not true: In (2.10) the chamber of the non-disecting reflection on $\mathbb{R} \mathbb{P}^{2}$ equals $\mathbb{R} \mathbb{P}^{2}$ and the generating reflection is not associated to a wall since $\mathbb{R P}^{2}$ has no boundary.

For Coxeter manifolds the converse statement is also true as the following theorem shows.

Theorem. Let $C$ be a Riemannian chamber.

Then to each admissible Coxeter equipment $G$ of $C$ there exists a smooth Riemannian manifold $\mathcal{U}(G, C)$ without boundary and a discrete subgroup $G$ of isometries which is generated by reflections such that $C$ is isometric to a chamber of $M$ which is also a Dirichlet domain.

If $C$ is connected then also $M$ is connected. If the equipment $G=G_{\text {univ }}$ is the universal one then $G$ is generated by disecting reflections and $\pi_{1}(\mathcal{U}(G, C))=$ $\pi_{1}(C) *_{e} G_{u n i v}$. In general we have an exact sequence:

$$
\{1\} \rightarrow \pi_{1}(C) *_{e} G_{\text {univ }} \rightarrow \pi_{1}(\mathcal{U}(G, C)) \rightarrow G_{\text {univ }} \rightarrow G \rightarrow\{1\} .
$$

Proof. We use first the universal equipment. Let $\left\{F_{i}\right\}$ be the set of all closures of walls of $C$. We construct first the group $G$, as follows. For each wall $F_{i}$ of $C$ we take a generator $s_{i}$ of $G$. Then $G=G_{\text {univ }}$ is the group generated by all $s_{i}$ and with relations $\left(s_{i} s_{j}\right)^{n_{i j}}=1$, when $F_{i} \cap F_{j} \neq \emptyset$ and where the angle between $F_{i}$ and $F_{j}$ is $\pi / n_{i j}$. For each $x \in C$ we constructed in (4.4) a linear Coxeter group $G_{x} \subset O\left(T_{x} C, g_{x}\right)$ which is generated by those $s_{i}$ for which $x \in F_{i}$. Obviously, $G_{x}$ is a subgroup of $G$.

Now we construct $M=\mathcal{U}(G, C)$ as topological space by putting $\mathcal{U}(G, C):=$ $G \times C / \sim$ where

$$
\begin{gathered}
\left(g . s_{i}, x\right) \sim\left(g, s_{i}(x)\right)=(g, x) \text { for } x \in F_{i}, \quad \text { or equivalently } \\
(g, x) \sim(h, y) \Longleftrightarrow x=y \text { and } g^{-1} h \in G_{x} .
\end{gathered}
$$

So $\mathcal{U}(G, C)$ is a quotient of the disjoint union of $|G|$ copies of $C$ which are glued together only along walls.

We construct an atlas for $\mathcal{U}(G, C)$ as follows, using the arguments from (4.4). For a corner $x$ of $C$ consider the Riemannian metric $\tilde{\gamma}_{B_{x}}$ on the open ball $B_{x} \subset T_{x} C$ which is smooth by condition (4.4.2), and the smooth exponential mapping $\exp _{x}$ : $V_{x}=B_{x} \cap Q_{x} \rightarrow W_{x} \subset C$. We extend it to a $G_{x}$-equivariant homeomorphism exp $\tilde{p}_{x}$ from $B_{x}$ to the open neighborhood $U_{x}=\bigcup_{g \in G_{x}}\left(\{g\} \times W_{x}\right)$ of $x$ in $M$ by putting $\exp _{x}(g \cdot X)=\left(g, \exp _{x}(X)\right)$ for $X \in V_{x}$ and $g \in G_{x}$. Then $\left(U_{x}, u_{x}:=\exp _{x}{ }^{-1}: U_{x} \rightarrow\right.$ $\left.B_{x} \in T_{x} C\right)$ is a chart on $M$.

If $x \in C$ is a regular point we use the inverse of the exponential mapping on such a small neigborhood of 0 in $T_{x} C$ that its image does not meet any wall. These charts we the distribute from $C=\{\operatorname{Id}\} \times C$ to the whole of $M$ by using the transformations from $g$.

We claim that this gives a smooth atlas for $\mathcal{U}(G, C)$ : Suppose that $x$ and $y$ are corners of $C$ such that $W_{x} \cap W_{y} \neq \emptyset$. We have to show that $u_{x} \circ u_{y}^{-1}$ is smooth. 
We may assume that $y \in W_{x}$ since we may connect $x$ and $y$ by finitely many chart changings with this property. But then this is a chart change of exponential mappings at different base points of the smooth Riemannian metric in $B_{x} \subset T_{x} C$.

Finally, $G$ acts on the smooth manifold $\mathcal{U}(G, C)$ by construction: $g \cdot\left(g_{1}, x\right)=$ $\left(g g_{1}, x\right)$, and it consists of isometries. By construction $G$ acts freely and transitively on the set of all chambers of $\mathcal{U}(C, G)$. We claim that the generators $s_{i}$ of $G$ are disecting. Suppose for contradiction that a generator $s$ is not disecting. Choose regular point $x_{0} \in C^{o}$ and a smooth curve $c$ in $\mathcal{U}(C, G) \backslash \mathcal{U}(C, G)^{s}$ from $x_{0}$ to s. $x_{0}$ which is transversal to all intersections of reflection hypersurfaces. Then $c$ passes from $C$ to a neighbor $s_{i_{1}} C$, then to a neighbor $s_{i_{1}} s_{i_{2}} C$ of $s_{i_{1}} C$, and so on, till it reaches the chamber $s_{i_{1}} \ldots s_{i_{k}} C=s C$ containing $s . x_{0}$. None of the $s_{i_{j}}$ equals $s$ since $c$ does not meet $\mathcal{U}(C, G)^{s}$. Since $G$ acts freely and transitively on the set of chambers we have $s=s_{i_{1}} \ldots s_{i_{k}}$ in $G$, a contradiction. (WHY??)

Finally, for a general admissible equipment we have a normal subgroup $R \subset G$ of further relations which by the description of an admissible equipment acts freely and discretely on the universal $M$ which thus is a covering of the resulting manifold. The statement on fundamental groups follows from (3.9).

4.6. Remark. We can also consider manifold with corners $C$ with a smooth Riemannian metric $g$ which satisfies only condition (4.4.1). Then we can construct a topological manifold $M$ which is smooth off the union of all reflection hypersurfaces, with a Riemannian metric which is only continuous along the the reflection hypersurfaces, in general. It might be worthwile to study this object.

4.7. Theorem. Let $C$ be a manifold with corners with a Coxeter equipment $s: W \mapsto s(W) \in S$ where $(G, S)$ is a Coxeter system.

Then there exists a Riemannian metric $\gamma$ such that $(C, \gamma)$ is a Riemannian chamber and $s$ is an admissible equipment for it.

Proof. We construct the metric inductively starting from faces which are manifolds without boundary. On each such face $F$ we put an arbitrary Riemannian metric $\gamma_{F}$.

Now let $F$ be a face which contains corners of index (in $F$ ) at most 1, i.e., $F$ is a manifold with boundary $\partial F$ which is a disjoint union of faces $F_{1}$ without boundary. Along each boundary component $F_{1}$ of $F$ we consider an open collar $F_{1} \times[0,1) \subset F$ and extend the metric by $\gamma(x, t)=d t^{2}+\gamma_{F_{1}}(x)$ where $x \in F_{1}$ and $t$ is the coordinate function on $[0,1)$. With a partition of unity we may extend this metric to the whole of $F$ in such a way that near each $F_{1}$ it is not changed. Note that $F_{1}$ is totally geodesic in $F$, and that the metric is constant in the direction $t$ normal to $F_{1}$.

Now let $F$ be a face which contains corners of index (in $F$ ) at most 2, i.e., $\partial F$ contains walls $F_{1}^{i}$ of $F$ which are manifolds with boundary. We already defined Riemannian metrics $\gamma_{F_{1}^{i}}$ on $F_{1}^{i}$. If $F_{2}$ is a boundary component of $F_{1}^{1} \cap F_{1}^{2}$ we consider an open tubular wedge neigbourhood $F_{2} \times D$ of $F_{2}$ in $F$ with the following property. Each fiber $\{x\} \times D$ intersects $F_{1}^{i}$ exactly in the fiber $\{x\} \times[0,1)$ of that collar of $F_{2}$ in $F_{1}^{i}$ for each $x \in F_{2}$ which was used above to construct the Riemannian metric on $F_{1}^{i}$. The fiber $D \subset \mathbb{R}^{2}$ is an open 0-neighborhood in a quadrant with angle $\alpha_{F}\left(F_{1}^{1}, F_{1}^{2}\right)$ as in fig. 9 . Here $\alpha_{F}\left(F_{1}^{1}, F_{1}^{2}\right)$ is determined by the Coxeter equipment: If in terms of walls $W_{i}$ of $C$ we have

$$
F=W_{i_{1}} \cap \cdots \cap W_{i_{n-2}},
$$




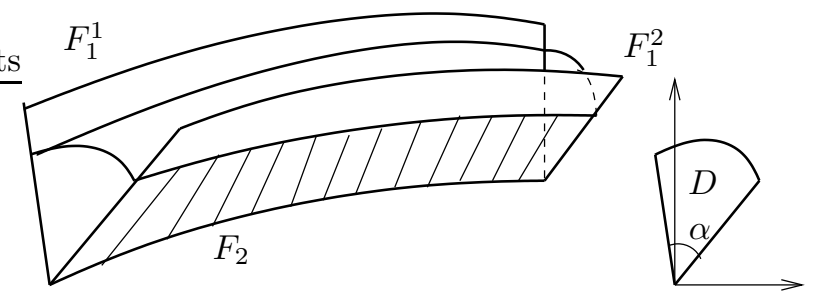

Figure 9. The tubular wedge neigborhood $F_{2} \times D$ and its fiber $D$.

$$
\begin{gathered}
F_{1}^{1}=W_{i_{1}} \cap \cdots \cap W_{i_{n-2}} \cap W_{i_{n-1}}, \quad F_{1}^{2}=W_{i_{1}} \cap \cdots \cap W_{i_{n-2}} \cap W_{i_{n}}, \\
F_{2}=W_{i_{1}} \cap \cdots \cap W_{i_{n-2}} \cap W_{i_{n-1}} \cap W_{i_{n}},
\end{gathered}
$$

then $\alpha_{F}\left(F_{1}^{1}, F_{1}^{2}\right)$ is determined by the (finite) Coxeter system $\left(G\left(F_{2}\right)=G\left(S^{\prime}\right), S^{\prime}\right)$ where $S^{\prime}=\left\{s_{i_{1}}, \ldots, s_{i_{n}}\right\}$, by considering the angle between and in the corresponding faces in the Weyl chamber of $\left(G\left(S^{\prime}\right), S^{\prime}\right)$, as decribed in (3.3).

We now put the product metric $\gamma_{D}(u)+\gamma_{F_{2}}(x)$ for $(x, u) \in F_{2} \times D$ on the tubular wedge neigbourhood $F_{2} \times D$, where $\gamma_{D}$ is the standard Euclidean metric on $\mathbb{R}^{2}$ restricted to $D$. This gives a metric on $F_{2} \times D$ which induces the already constructed metric $\gamma_{F_{1}^{i}}$ on the intersection with $F_{1}^{i}$ since $F_{2} \times D$ intersects $F_{1}^{i}$ in the collar used to construct $\gamma_{F_{1}^{i}}$. Moreover $F_{2}$ and the parts of $F_{1}^{i}$ are totally geodesic, and the metric is constant in directions normal to any relevant face, near that face.

We do this construction near any face of codimension 2 of $F$. Then we use a collar $\left(F_{1}^{i} \backslash \partial F_{1}^{i}\right) \times[0,1)$ of the interior of the face $F_{1}^{i}$ in $F$ such that the fiber near any $F_{2}$ coincides with the normal geodesic in $F_{2} \times D$ in the metric constructed there. Put the metric $d t^{2}+\gamma_{F_{1}^{i}}(x)$ for $(x, t) \in\left(F_{1}^{i} \backslash \partial F_{1}^{i}\right) \times[0,1)$ on this collar, and use a partition of unity on the union of all these collars and the wedge neighborhoods which is constant in the normals near any face to glue the metrics in such a way that the resulting metric is constant in the normal directions near any face and each face is totally geodesic. With another partition of unity we extend this metric into the interior of $F$ and not changing it near any face.

We proceed inductively. We assume that we have already constructed in this way metrics on each face which consists of corners of index $\geq k$ in $C$ and consider now a face $F$ which consists of corners of index $\geq k-1$ in $C$. Then the boundary $\partial F$ is a union of faces where we alredy constructed the metric. Let $F_{k}$ be a minimal face in $\partial F$, i.e., $F_{k}$ does not contain any other face. Then $F_{k}$ is a manifold without boundary where we already have a metric $\gamma_{F_{k}}$. Moreover $F_{k}$ is the transversal intersection of $k$ walls $F_{1}^{1}, \ldots, F_{1}^{k}$ of $F$, where $k$ is the codimension of $F_{k}$ in $F$. We then choose a tubular wedge neighbourhood $F_{k} \times D^{k}$ of $F_{k}$ in $F$ which intersects fiber respectingly each intersection of $k-1$ of the walls $F_{1}^{1}, \ldots, F_{1}^{k}$ of $F$ in the tubular wedge neighborhood which was used previously to construct the metric $\gamma_{F_{1}^{i}}$ on each of the walls. Here $D^{k}$ is an open 0 -neighborhood in a quadrant in $\mathbb{R}^{k}$ with walls whose angles $\alpha_{F}\left(F_{1}^{i}, F_{1}^{j}\right)$ are determined by the Coxeter equipment as described above. We now put the metric $\gamma_{D^{k}}(u)+\gamma_{F_{k}}(x)$ for $(x, u) \in F_{k} \times D^{k}$ on the tubular wedge neigbourhood $F_{k} \times D^{k}$, where $\gamma_{D^{k}}$ is the standard Euclidean metric on $\mathbb{R}^{k}$ restricted to $D^{k}$. This gives a metric on $F_{k} \times D^{k}$ which induces the already constructed metric $\gamma_{F_{1}^{i}}$ on the intersection with $F_{1}^{i}$ since $F_{k} \times D^{k}$ intersects $F_{1}^{i}$ in the tubular wedge neighborhood used to construct $\gamma_{F_{1}^{i}}$. Moreover $F_{k}$ and the 
parts of $F_{1}^{i}$ are totally geodesic, and the metric is constant in directions normal to any face near that face.

We do this construction near any minimal face of $F$. Then we use a collar $\left(F_{1}^{i} \backslash \partial F_{1}^{i}\right) \times[0,1)$ of the interior of the face $F_{1}^{i}$ in $F$ such that the fiber near any minimal face $F_{l}$ coincides with the normal geodesic in $F_{l} \times D^{l}$ in the metric constructed there. Put the metric $d t^{2}+\gamma_{F_{1}^{i}}(x)$ for $(x, t) \in\left(F_{1}^{i} \backslash \partial F_{1}^{i}\right) \times[0,1)$ on this collar, and use a partition of unity on the union of all these collars and the wedge neighborhoods which is constant in the normals near any face, to glue the metrics in such a way that the resulting metric is constant in the normal directions near any face and each face is totally geodesic. With another partition of unity we extend this metric into the interior of $F$.

Eventually we exhaust each connected component of $C$.

4.8. Proposition. Let $C$ be a manifold with corners with a Coxeter equipment $s$ : $W \mapsto s(W) \in S$ where $(G, S)$ is a Coxeter system. Let $\gamma$ and $\gamma^{\prime}$ be two Riemannian metrics on $C$ such that $(C, \gamma)$ and $\left(C, \gamma^{\prime}\right)$ are both Riemannian chambers and $s$ is an admissible equipment for both.

Then the smooth manifolds $\mathcal{U}(G, C, \gamma)$ and $\mathcal{U}\left(G, C, \gamma^{\prime}\right)$ constructed via (4.5) are diffeomorphic.

Proof. Since the construction as a topological space described in the proof of (4.5) depends only on the equipment, the two manifolds are canonically homeomorphic. For a corner $x \in C$ let $u_{x}: U_{x} \rightarrow B_{x} \subset T_{x} C$ and $u_{x}^{\prime}: U_{x}^{\prime} \rightarrow B_{x} \subset T_{x} C$ be two charts as described in the proof of (4.5) for the two Riemannian metrics $\gamma$ and $\gamma^{\prime}$. But then the chart change $u_{x}^{\prime} \circ u_{x}^{-1}$, considered in a manifold without boundary which contains $C$ as a submanifold with corners (see (4.1)), consists of the exponential mapping of the extended Riemannian metric $\tilde{\gamma}$ followed by the inverse of the exponential mapping of $\tilde{\gamma}^{\prime}$, which is obviously smooth. Thus the canonical homeomorphism between $\mathcal{U}(G, C, \gamma)$ and $\mathcal{U}\left(G, C, \gamma^{\prime}\right)$ is a diffeomorphism.

\section{ORBIFOLDS}

5.1. Smooth orbifolds. We recall the definition of orbifold. Let $X$ be a second countable Hausdorff space. An atlas of a smooth $n$-dimensional orbifold (or $V$ manifold) on $X$ is a family $\left\{U_{i}\right\}_{i \in I}$ of open sets that satisfy:

(1) $\left\{U_{i}\right\}_{i \in I}$ is an open cover of $\mathrm{X}$.

(2) For each $i \in I$ a local uniformizing system consisting of a triple $\left\{\tilde{U}_{i}, G_{i}, \varphi_{i}\right\}$, where $\tilde{U}_{i}$ is a connected open subset of $\mathbb{R}^{n}$ containing the origin, $G_{i}$ is a finite group of diffeomorphisms acting effectively and properly on $\tilde{U}_{i}$, and $\varphi_{i}: \tilde{U}_{i} \rightarrow U_{i}$ is a continuous map of $\tilde{U}_{i}$ onto $U_{i}$ such that $\varphi_{i} \circ g=\varphi_{i}$ for all $g \in G_{i}$ and the induced map of $\tilde{U}_{i} / G_{i}$ onto $U_{i}$ is a homeomorphism. The finite group $G_{i}$ is called a local uniformizing group.

(3) Given $\tilde{x}_{i} \in \tilde{U}_{i}$ and $\tilde{x}_{j} \in \tilde{U}_{j}$ such that $\varphi_{i}\left(\tilde{x}_{i}\right)=\varphi_{j}\left(\tilde{x}_{j}\right)$, there is a diffeomorphism $\varphi_{i j}: \tilde{V}_{j} \rightarrow \tilde{V}_{i}$ from a neighborhood $\tilde{V}_{i} \subset \tilde{U}_{i}$ of $\tilde{x}_{i}$ onto a neighborhood $\tilde{V}_{j} \subset \tilde{U}_{j}$ of $\tilde{x}_{j}$ such that $\varphi_{i}=\varphi_{j} \circ \varphi_{j i}$.

Two atlases are equivalent if their union is again an atlas of a smooth orbifold on $X$. An orbifold is the space $X$ with an equivalence class of atlaces of smooth orbifolds on $X$. 
Proposition. [31] If $M$ is an $n$-dimensional smooth manifold and $G$ is a group acting smoothly and discretely on $M$, then $X=M / G$ has a structure of orbifold.

Proof. Let $x \in X$. Choose $\tilde{x} \in M$ projecting to $x$, and denote by $G_{x}$ the isotropy group of $\tilde{x}$. Choose a neigborhood of $\tilde{U}_{x}$ invariant by $G_{x}$ and disjoint from $g\left(U_{x}\right)$ for all $g \in G \backslash G_{x}$ such that there is a local chart $k: U_{x} \rightarrow \tilde{U}_{x} \subset \mathbb{R}^{n}$ on $M$ with $k(x)=0$. We take $\left(\tilde{U}_{x}, G_{x}, \varphi_{x}\right)$, where $\varphi_{x}$ is a composition of $k^{-1}$ with the projection $\tilde{U} \rightarrow \tilde{U} / G_{x}$, for a local uniformizing system. It is easily checked such local uniformizing systems form an atlas of a smooth $n$-dimensional orbifold on $M / G$.

In the definition of atlas of a smooth orbifold on $X$ we can always take the finite subgroups $G_{i}$ to be subgroups of the orthogonal group $O(n)$ acting naturally on $\mathbb{R}^{n}$. Condition (3) implies that for each $g_{i} \in G_{i}$ there exists $g_{j} \in G_{j}$ such that $\varphi_{j i} \circ g_{i}=g_{j} \circ \varphi_{j i}$.

Let $\left\{\tilde{U}_{i}, G_{i}, \varphi_{i}\right\}$ be a unifomizing system such that $\tilde{U}_{i}$ contains the origin, the group $G_{i}$ is a subgroup of $O(n)$, and $x=\varphi_{i}(0)$. Then the group $G_{x}=G_{i}$ is independent of the uniformizing system $\left\{\tilde{U}_{i}, G_{i}, \varphi_{i}\right\}$. More precisely, this group is defined up to isomorphism and its action on $\mathbb{R}^{n}$ is defined up to isomorphism as well. The point $x \in X$ is called regular if the corresponding group $G_{x}$ is trivial and otherwise singular.

5.2. Reconstruction of the orbifold structure from the structure sheaf. Let again $\left\{\tilde{U}_{i}, G_{i}, \varphi_{i}\right\}$ be a unifomizing system such that $\tilde{U}_{i}$ contains the origin, the group $G_{i}$ is a subgroup of $O(n)$, and $x=\varphi_{i}(0)$. Then there is a representation $\rho: G_{i} \rightarrow O(n)$, a ball $B$ in $\mathbb{R}^{n}$ centered at the origin, and a map $\varphi: B \rightarrow X$ such that $\varphi(0)=x$ and $\left\{B, G_{i}, \varphi\right\}$ is a uniformizing system of the orbifold $X$.

A function $f: U_{i} \rightarrow \mathbb{R}$ is called smooth if $f \circ \varphi_{i}$ is a smooth function on $\tilde{U}$. The germs of smooth functions on $X$ define a sheaf $S_{X}$ on $X$.

5.3. Definition. Let $X$ and $\tilde{X}$ be two smooth orbifolds. The orbifold $\tilde{X}$ is called a covering orbifold for $X$ with a projection $p: \tilde{X} \rightarrow X$ if $p$ is a continuous map of underlying topological spaces and each point $x \in X$ has a neighborhood $U=\tilde{U} / G$ (where $\tilde{U}$ is an open subset of $\mathbb{R}^{n}$ ) for which each component $V_{i}$ of $p^{-1}(U)$ is isomorphic to $\tilde{U} / G_{i}$, where $G_{i} \subset G$ is some subgroup. The above isomorphisms $U=\tilde{U} / G$ and $V_{i}=\tilde{U} / G_{i}$ must respect the projections.

Note that the projection $p$ in the above definition is not a cover of underlying topological spaces.

Hereafter we suppose that all orbifolds and their covering orbifolds are connected.

5.4. Theorem. [31] An orbifold $X$ has a universal covering orbifold $p: \tilde{X} \rightarrow X$. More precisely, if $x \in X, \tilde{x} \in \tilde{X}$ are regular points and $p(\tilde{x})=x$, for any other covering orbifold $p^{\prime}: \tilde{X}^{\prime} \rightarrow X$ and $\tilde{x}^{\prime} \in \tilde{X}^{\prime}$ such that $p^{\prime}\left(\tilde{x}^{\prime}\right)=x$ there is a cover $q: \tilde{X} \rightarrow \tilde{X}^{\prime}$ such that $p=p^{\prime} \circ q$ and $q(\tilde{x})=\tilde{x}^{\prime}$. For any points $\tilde{x}, \tilde{x}^{\prime} \in p^{-1}(x)$ there is a deck transformation of $\tilde{X}$ taking $\tilde{x}$ to $\tilde{x}^{\prime}$.

Suppose $\rho: G \rightarrow O(n)$ is a representation of a finite group $G, \mathbb{R}^{n} / G$ is the corresponding orbifold, and $S_{\mathbb{R}^{n} / G}$ is the corresponding sheaf. By the Hilbert theorem the ring $\mathbb{R}\left[\mathbb{R}^{n}\right]^{G}$ is finitely generated. Let $\sigma^{1}, \ldots, \sigma^{m}$ be a system of homogeneous generators of $\mathbb{R}\left[\mathbb{R}^{n}\right]^{G}$ and $y^{1}, \ldots, y^{m}$ the corresponding functions on $\mathbb{R}^{n} / G$. Consider the map $\sigma=\left(\sigma^{1}, \ldots, \sigma^{m}\right): \mathbb{R}^{n} \rightarrow \mathbb{R}^{m}$ called the orbit map. It is known 
[2] that the map $\sigma$ induces a homeomorphism between $\sigma\left(\mathbb{R}^{n}\right)$ and the orbit space $\mathbb{R}^{n} / G$ which establishes an isomorphism between the restriction of the sheaf $C_{m}^{\infty}$ of smooth functions on $\mathbb{R}^{m}$ to $\sigma\left(\mathbb{R}^{n}\right)$ and the sheaf $S_{\mathbb{R}^{n} / G}$.

It is clear that for each orbifold $X$ and $x \in X$ there is a neghborhood $U_{x}$ and a representation $\rho: G_{x} \rightarrow O(n)$ such that the restriction of $S_{X}$ to $U_{x}$ is isomorphic to the restriction of the sheaf $S_{\mathbb{R}^{n} / G_{x}}$ to some ball centered at the origin.

For a representation $\rho: G \rightarrow O(n)$ a diffeomorphism of the orbit space $\mathbb{R}^{n} / G$ is an automorphism of the sheaf $S_{\mathbb{R}^{n} / G}$ by definition. Let $f: \mathbb{R}^{n} / G \rightarrow \mathbb{R}^{n} / G$ be a diffeomorphism and $h^{1}, \ldots, h^{m}$ a system of generators of $S_{\mathbb{R}^{n} / G}$. Then $f$ is uniquely defined by the images of generators $h^{i}$ and these images are the generators of $S_{\mathbb{R}^{n}} / G$ again. Denote by $R$ the set of all reflections contained in $G$ and by $A(G, R)$ the set of all automorphisms of the group $G$ which preserves the set $R$.

5.5. Theorem. [23] For each diffeomorphism $f$ of the orbit space $\mathbb{R}^{n} / G$ there is a smooth lift $F: \mathbb{R}^{n} \rightarrow \mathbb{R}^{n}$. For each such lift $F$ there is an automorphism $a \in A(G, R)$ such that for all $g \in G$ and $x \in \mathbb{R}^{n} / G$ we have $F(g x)=a(g) F(x)$.

The local version of this theorem is also true, i.e. if $B$ is a ball in $\mathbb{R}^{n}$ centered at the origin and $f$ is a diffeomorphism of the sheaf $S_{B / G}$, then there is a smooth lift $F: B \rightarrow B$ with the same property as above.

5.6. Theorem. An orbifold $X$ is defined uniquely by its sheaf $S_{X}$.

Proof. Note that for a regular point $x \in X$ the ring $S_{X}(x)$ of the germs of $S_{X}$ at $x$ is isomorphic to the ring of germs at 0 of smooth functions on $\mathbb{R}^{n}$. Then the dimension of the orbifold $X$ is defined by the sheaf $S_{X}$. Next note that if $\rho: G \rightarrow O(n)$ is a representation of a finite group $G$, then the group preserving all smooth $G$-invariant functions on $\mathbb{R}^{n}$ coincides with $\rho(G)$. If this group is infinite there is a regular point with non trivial stabilizer, which is impossible. The result then follows from the fact that the order of $G$ equals the cardinality of a regular orbit.

It is sufficient to prove that for each a finite group $G$, a representation $G \rightarrow O(n)$, a ball $B$ in $\mathbb{R}^{n}$, and the map $\varphi: B \rightarrow X$ which induces an isomorphism of the sheaf $S_{B / G}$ and the restriction $S_{U}$ of the sheaf $S_{X}$ to some open subset $U$ of $X,\{B, G, \varphi\}$ is a uniformizing system on $X$.

Let $\left\{B_{1}, G_{1}, \varphi_{1}\right\}$ be such a uniformizing system, corresponding to the representation $\rho_{1}: G_{1} \rightarrow O(n), \varphi(0)=x$, and $\left\{B_{2}, G_{2}, \varphi_{2}\right\}$ a uniformizing system of the orbifold $X$ which is induced by some representation $\rho_{2}: G_{2} \rightarrow O(n)$ such that $\varphi_{2}(0)=x$. We may assume that $B_{1}=B_{2}=B$ and $\varphi_{1}(B)=\varphi_{2}(B)=U$. Then the rings of functions on $B$ which are compositions of $\varphi_{1}$ and $\varphi_{2}$ with the sections of $S_{X}$ on $U$ coincides. By the above remark $\rho_{1}\left(G_{1}\right)=\rho_{2}\left(G_{2}\right)=G$.

For $i=1,2$ denote by $\bar{\varphi}_{i}$ the diffeomorphism $B / G \rightarrow U$ induced by $\varphi_{i}$. Then $\bar{\varphi}_{2}^{-1} \circ \bar{\varphi}_{1}$ is a diffeomorphism of $B / G$. By Theorem (5.5) there is a smooth lift $B \rightarrow B$ of this diffeomorphism. But this means that $\left\{B_{1}, G_{1}, \varphi_{1}\right\}$ is a uniformizing system of the orbifold $X$.

5.7. Corollary. Let a group $G$ acts discretely on a smooth simply connected manifold $M$ and $S_{X}$ the corresponding sheaf on $X=M / G$. Then $M$ is a universal covering orbifold for $X$.

Proof. Evidently manifold $M$ is a covering orbifold for $X$. If $\tilde{X}$ is universal covering orbifold for $X$, then there is a cover $q: \tilde{X} \rightarrow M$. By the definition 
of cover $\tilde{X}$ should be a manifold and $q$ a cover of manifilds. Therefore $q$ is a diffeomorphism.

Theorems (5.6) and (5.7) imply the following statement.

5.8. Corollary. Let a group $G$ act discretely on a smooth simply connected manifold $M$ and $S_{X}$ the corresponding sheaf on $X=M / G$. Then each diffeomorphism of the orbit space $X$, i.e. an automorphism of the sheaf $S_{X}$ has a smooth lift to $M$.

\section{Coxeter orbifold}

6.1. Coxeter orbifolds. A smooth orbifold $X$ is called a Coxeter orbifold if for each local uniformizing system $\left(\tilde{U}_{i} \subset \mathbb{R}^{n}, G_{i} \subset O(n), \varphi_{i}\right)$ the group $G_{i}$ is a finite linear Coxeter group.

6.2. Example. Let $M$ be a Coxeter Riemannian manifolds with reflection group $G$. Then any Weyl chamber is a Coxeter orbifold. This follows from proposition (5.1) and (2.11).

6.3. Coxeter orbifold as a manifold with corners and its universal Coxeter equipment. Let $X$ be a Coxeter orbifold. Let $\left(\tilde{U}_{i}, G_{i}, \varphi_{i}\right)_{i \in I}$ be an atlas of local uniformizing systems on $X$ such that $\left(U_{i}\right)$ is an open cover of $X$. Then $\tilde{U}_{i} \subset \mathbb{R}^{n}$ is an open neighborhood of 0 which is invariant under the Coxeter group $G_{i}$. Thus the orbit space $\tilde{U}_{i} / G_{i}$ is an open neighborhood of 0 in a linear Weyl chamber of the group $G_{i}$. The (equivariant) chart changings $\varphi_{i j}$ induce smooth chart changings between open subsets of $\tilde{U}_{j} / G_{j}$ and $\tilde{U}_{i} / G_{i}$. These respect the indices of corners (see (4.1)). Thus they describe a smooth atlas for the structure of a manifold with corners on $X$. So walls and faces are defined and to each wall $W$ one can associate a generator $s(W)$ of the Coxeter system with the following property: If $W \cap U_{i} \neq \emptyset$ for a local uniformizing system $\left(\tilde{U}_{i}, G_{i}, \varphi_{i}\right)$, then $s(W)$ equals the generator of $G_{i}$ which is given by the reflection in the wall $\varphi_{i}^{-1}(W) \subset \tilde{U}_{i}$. Then $\left(s(W) s\left(W^{\prime}\right)\right)^{n\left(W, W^{\prime}\right)}=1$ if $\varphi_{i}^{-1}(W), \varphi_{i}^{-1}\left(W^{\prime}\right) \neq \emptyset$ the generators corresponding to them in $G_{i}$ satisfy the same relation.

6.4. Theorem. Any Coxeter orbifold is the Weyl chamber of a Riemannian Coxeter manifold.

Proof. This follows from (6.3) and (4.7).

6.5. Corollary. Any Coxeter orbifold is good in the sense of Thurston [31].

6.6. Coxeter orbifold structures on a simplex. Let $\Delta_{n}$ be the standard $n$ simplex with vertices $0,1, \ldots, n$. If $s$ is a Coxeter $(G, S)$-equipment of $\Delta_{n}$, then there exist a Riemannian metric $\gamma$ on $\Delta_{n}$, such that $\left(\Delta_{n}, \gamma\right)$ is a Riemannian chamber and the equipment $s$ is admissible. We denote by $M=\mathcal{U}\left(G, \Delta_{n}, s, \gamma\right)$ the associated Coxeter $G$-manifold. It is simply connected. The homeomorphism $M / G \cong \Delta_{n}$ define on $\Delta_{n}$ a structure of Coxeter orbifold, with the universal covering manifold $M$, which depends only on the equipment $s$, by (4.8). Hence, a description of Coxeter orbifold structures on $\Delta_{n}$ and also Coxeter $G$-manifolds with the orbit space $\Delta_{n}$ up to a $G$-diffeomorphism reduces to a description of Coxeter equipments of $\Delta$.

For any finite Coxeter group $G$ with the generators $S=\left\{s_{0}, \ldots, s_{n}\right\}$ there exist a unique natural equipment such that the wall $W_{i}=(0,1, \ldots, \widehat{i}, \ldots, n)$ corresponds to $s_{i}$ for $i=0, \ldots, n$. The corresponding Coxeter manifold $M$ is the sphere $S^{n}$ with 
the natural action of $G$ induced by the standard representation of $G$ in $\mathbb{R}^{n+1}$. Let now $G$ be an infinite Coxeter group with system of generators $S$. There exists a Coxeter $(G, S)$-equipment of $\Delta_{n}$ if and only if $|S|=n+1$ and the Coxeter subgroup generated by $S \backslash\{s\}$ is finite for any $s \in S$. In term of the Coxeter diagram $\Gamma$ of the group $G$, this means that all connected components of $\Gamma$ with exception of one component $\Gamma_{0}$ correspond to finite Coxeter groups, and the component $\Gamma_{0}$ corresponds to an infinite Coxeter group, but after deleting any node it become a Coxeter diagram of a finite Coxeter group. One can easily check that such a Coxeter diagram $\Gamma_{0}$ is either a connected parabolic Coxeter diagram (i.e., extended Dynkin diagram of a simple Lie algebra) or one of the following diagrams:

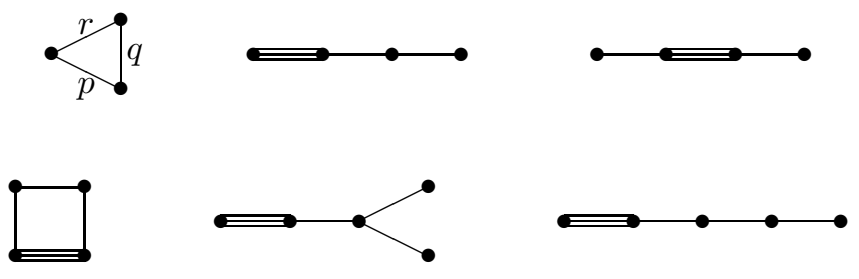

An interesting question is to classify such equipments for other polyhedra, e.g., a cube, prism etc.

May be, it is possible to construct a non trivial example of compact Coxeter manifolds, e.g. simply connected 3-manifolds.

\section{REFERENCES}

1. Dmitri Alekseevky, Andreas Kriegl, Mark Losik, Michor, and Peter W., Lifting smooth curves over invariants for representations of compact Lie groups, Transformation groups 5 (2000), no. 2, 103-110. arXiv:math.DG/9801029.

2. _ The Riemannian geometry of orbit spaces. The metric, geodesics, and integrable systems, Publ. Math. 62 (2003), no. 3-4, arXiv:math.DG/0102159.

3. E. Bierstone, Lifting isotopies from orbit spaces, Topology 14 (1975), 245-252.

4. N. Bourbaki, Groupes et algèbres de Lie, Hermann, Paris, 1968, Ch. 4-6.

5. L. Bröcker, Semialgebraische Geometrie, Jber. d. Dt. Math.-Verein. 97 (1995), 130-156.

6. Yu. Burago, M. Gromov, and G. Perel'man, A. D. Alexandrov spaces with curvature bounded below, Russ. Math. Surv. 47 (1992), 1-58.

7. Ruth Charney and Michael W. Davis, Singular metrics of nonpositive curvature on branched covers of Riemannian manifolds, Am. J. Math. 115 (1993), 929-1009.

8. The Euler characteristic of a nonpositively curved, piecewise Euclidean manifold, Pac. J. Math. 171 (1995), 117-137.

9. groups, J. Am. Math. Soc. 8 (1995), 597-627.

10. Yuan-Jen Chiang, Spectral geometry of v-manifolds and its application to harmonic maps, Differential geometry. Part 1: Partial differential equations on manifolds, Proc. Symp. Pure Math. 54, Part 1, 1993, pp. 93-99.

11. H.S.M. Coxeter, Discrete groups generated by reflections, Ann. Math. 35 (1934), 388-621

12. Michael W. Davis, Groups generated by reflections and aspherical manifolds not covered by Euclidean space, Ann. Math. 117 (1983), 293-324.

13. Coxeter groups and aspherical manifolds, Algebraic Topology, Aarhus 1982, Lect. Notes Math. 1051, Springer-Verlag, 1984, pp. 197-221.

14. — A hyperbolic 4-manifold, Proc. Am. Math. Soc. 93 (1985), 325-328.

15. 97-104.

16. Some aspherical manifolds, Duke Math. J. 55 (1987), 105-139.

17. $\underset{417-455}{ }$, Convex polytopes, Coxeter manifolds, and torus actions, Duke Math. J. 62 (1991), 
18. A. Douady and L. Hérault, Arrondissement des Variétés à coins, Comm. Math. Helv. 48 (1973), 484-491, Appendice à: A. Borel, J.P. Serre: Corners and arithmetic groups.

19. J. E. Humphreys, Reflection groups and Coxeter groups, Cambridge University Press, Cambridge, 1990, 1992, Cambridge studies in advanced mathematics 29.

20. N. Iwahori, On discrete reflection groups on symmetric Riemannian manifolds, pp. 57-62, Nippon Hyoronsha, Tokyo, 1966 w, MR 36\#830, Z 146.43501.

21. R. Kane, Reflection groups and invariant theory, Springer-Verlag, 2001, CMS books in Math. 5.

22. S Łojasiewicz, Triangulation of semi-analytic sets, Ann. Sc. Norm. Sup. Pisa, ser. III 18 (1964), 449-473.

23. M.V. Losik, Lifts of diffeomorphisms of orbit spaces for representations of compact Lie groups, Geom. Dedicata 88 (2001), 21-36.

24. M.A. Michailowa, Factor space under the action of a finite group generated by pseudo reflections, Izwest. Acad. Nauk USSR, Ser. Math. 48 (1984), 104-126.

25. Peter W. Michor, Manifolds of differentiable mappings, Shiva Publ., Orpington, 1980, Shiva Mathematics Series 3 MR 83g:58009, ZB 433.58001.

26. P.W. Michor, Isometric actions of Lie groups and invariants, Lecture course at the University of Vienna, 1996/97, www. univie.ac.at/ michor/tgbook.ps

27. Satake, Gauss-bonnet theorem for v-manifolds, J. Math. Soc. Japan 9 (1957), 464-492.

28. G. W. Schwarz, Smooth functions invariant under the action of a compact Lie group, Topology 14 (1975), 63-68.

29. ㄴ Lifting smooth homotopies of orbit spaces, Publ. Math. IHES 51 (37-136), 1980.

30. J. Szenthe, A significant property of real hyperbolic spaces, pp. 153-161, Babeş-Bolyai University, Cluj-Napoca, 1993, Preprint, 93-2 96b:53063.

31. William P. Thurston, Three-dimensional geometry and topology. Vol. 1., silvio levy, ed., Princeton University Press, Princeton, 1997, Princeton Mathematical Series 35.

32. E. B. Vinberg, Discrete linear groups that are generated by reflections, Izv. Akad. Nauk SSSR Ser. Mat. 35 (1971), 1072-1112, English translation, Math. USSR, Izv., 5 (1971), 1083-1119.

33. E.B. Vinberg, Discrete reflection groups in Lobachevsky spaces, Proc. Int. Congr. Math., Warszawa 1983, Vol. 1, 1984, pp. 593-601.

34. _ Hyperbolic reflection groups, Russ. Math. Surv. 40 (1985), 31-75.

35. E.B. Vinberg and O.V. Shvartsman, Discrete groups of motions of spaces of constant curvature, Geometry. II: Spaces of constant curvature, Encycl. Math. Sci. 29 (Berlin), SpringerVerlag, 1993, pp. 139-248.

36. H. Whitney, Elementary structure of real algebraic varieties, Ann. Math. 66 (1957), 545-556.

D.V. Alekseevsky: Department of Mathematics, University of Hull, Cottingham RoAd, Hull, HU6 7RX, England.

E-mail address: d.v.alekseevsky@maths.hull.ac.uk

A. Kriegl: Institut für Mathematik, Universität Wien, Strudlhofgasse 4, A-1090 WiEn, Austria.

E-mail address: Andreas.Kriegl@univie.ac.at

M. Losik: Saratov State University, ul. Astrakhanskaya, 83, 410026 Saratov, Russia.

E-mail address: LosikMV@info.sgu.ru

P. W. Michor: Institut für Mathematik, Universität Wien, Strudlhofgasse 4, A-1090 Wien, Austria; and: Erwin Schrödinger Institute of Mathematical Physics, BoltzmanNGasse 9, A-1090 Wien, Austria.

E-mail address: Peter.Michor@esi.ac.at 